\title{
Hydrologic response of diverse western rangelands
}

\author{
F.B. PIERSON, K.E. SPAETH, M.A. WELTZ, AND D.H. CARLSON
}

Authors are Research Hydrologist, United States Department of Agriculture (USDA), Agricultural Research Service (ARS), Northwest Watershed Research Center, Boise, Idaho; Rangeland Hydrologist, USDA, Natural Resources Conservation Service (NRCS), Northwest Watershed Research Center, Boise, Ida; Research Hydrologist, USDA, ARS, Great Plains System Research, Fort Collins, Colo.; and Support Scientist, University of Idaho, Department of Biological and Agricultural Engineering, Moscow, Ida.

\section{Abstract}

There are several generalizations or assumptions concerning rangeland hydrology and erosion relationships found in the literature and in the management arena. These generalizations have found their way into rangeland models, where modelers have assumed that diverse rangeland types can be lumped or averaged together in some way to develop one algorithm or equation to describe a process or relationship across the entire spectrum of rangeland types. These assumptions and modeling approaches based on the universal concept may not be appropriate for diverse rangeland types. This paper presents a comprehensive data set of vegetation, soils, hydrology, and erosion relationships of diverse western rangelands, and utilizes the data to assess the validity of the various assumptions/generalizations for rangelands. The data set emphasizes the difficulty in understanding hydrologic responses on semiarid rangelands, where the relationship between plant/soil characteristics and infiltration/erosion is not well established. When all sites were pooled together, infiltration and sediment production were not correlated with any measured vegetation or soil characteristic. A myriad group of factors determine infiltration and erosion, and is dependent on rangeland type and site conditions. The infiltration and erosion responses and correlation/regression analyses presented highlight the risk of using generalized assumptions about rangeland hydrologic response and emphasize the need to change the current modeling approach. Universal algorithms to represent the response of all rangeland types, such as the pooled multiple regression equations presented, will not provide sufficient accuracy for prediction or assessment of management. We need to develop a rationale to organize rangeland types/vegetation states according to similarities in relationships and responses. These functional rangeland units would assist in the development of more accurate predictive equations to enhance model performance and management of rangelands.

Key Words: rangeland hydrology, infiltration, erosion, hydrologic modeling

Rangeland ecosystems are comprised of diverse combinations of vegetation, soils, and climatic conditions. Rangeland managers need an understanding of this complexity to assess system health and apply appropriate management practices. Where direct knowledge of the rangeland system is limited, management decisions are often based on assumptions about how the system may

Manuscript accepted 16 Mar. 02

\section{Resumen}

Hay varias generalizaciones o supuestos concernientes a la hidrología de pastizales y las relaciones de erosión encontradas en la literatura y en la área de manejo. Estas generalizaciones han encontrado su camino dentro de los modelos de pastizales, donde los modeladores han asumido que diversos tipos de pastizales pueden ser agrupados o promediados juntos en alguna manera para desarrollar un algoritmo o ecuación para describir un proceso o relación a lo largo de un espectro de tipos de pastizal. Estas suposiciones o métodos de modelaje basados en un concepto universal pueden no ser apropiados para diversos tipos de pastizales. Este artículo presenta un juego de datos comprensivo de vegetación, suelos, hidrología y relaciones de erosión de diversos pastizales del oeste y utiliza los datos para evaluar la validez de varios supuestos/generalizaciones para los pastizales. El juego de datos enfatiza la dificultad en entender la respuesta hidrológica de los pastizales semiáridos, donde la relación entre las características de planta/suelo e infiltración/erosión no están bien establecidas. Cuando todos los sitios se agruparon, la infiltración y la producción de sedimento no estuvieron correlacionados con ninguna de las características del suelo o planta medidas. Un grupo indeterminado de factores determina la infiltración y erosión y es dependiente del tipo de pastizal y condiciones del sitio. Las respuestas de infiltración y erosión y los análisis de correlación/regresión presentan en forma destacada el riesgo de usar suposiciones generalizadas acerca de la respuesta hidrológica de los pastizales y enfatiza la necesidad de cambiar los métodos actuales de modelaje. Los algoritmos universales para representar la respuesta de todos los tipos de pastizal, tal como se fusionaron en las ecuaciones de regresión múltiple presentadas, no proveerán suficiente certeza para la predicción o evaluación del manejo. Necesitamos desarrollar un fundamento para organizar los estados de tipos de pastizal/vegetación de acuerdo a similitudes en relaciones y respuestas. Estas unidades funcionales de pastizal asistirán en desarrollar ecuaciones predictivas más certeras para mejorar el modelo de comportamiento y manejo de los pastizales.

work. Some of the generalizations or assumptions concerning rangeland hydrology and erosion relationships found in the literature are:

1. Rangeland ecological status/similarity index is directly related to hydrologic condition (Ellison 1949, Osborn 1952),

2. Sediment production is highly correlated with amount of infiltration/runoff (Blackburn and Skau 1974, Buckhouse 1984), 
3. Quantity of plant material and litter is positively correlated with infiltration (Gifford 1985, Wilcox et al. 1988),

4. Brush management alters hydrology and erosion (Bedunah and Sosebee 1985, Carlson et al.1990),

5. Vegetation characteristics dominate rangeland hydrology response (Branson et al. 1981, Stoddart et al. 1975, USDA-NRCS 1997).

While numerous examples can be found in the literature to further support these assumptions, little is known about the universality of such assumptions to all rangelands.

This concept of universality has found its way into rangeland models that managers use to assist in the assessment and management of rangelands. Modelers frequently use simplifying assumptions or generalizations to reduce the complexity of model design and system representa-

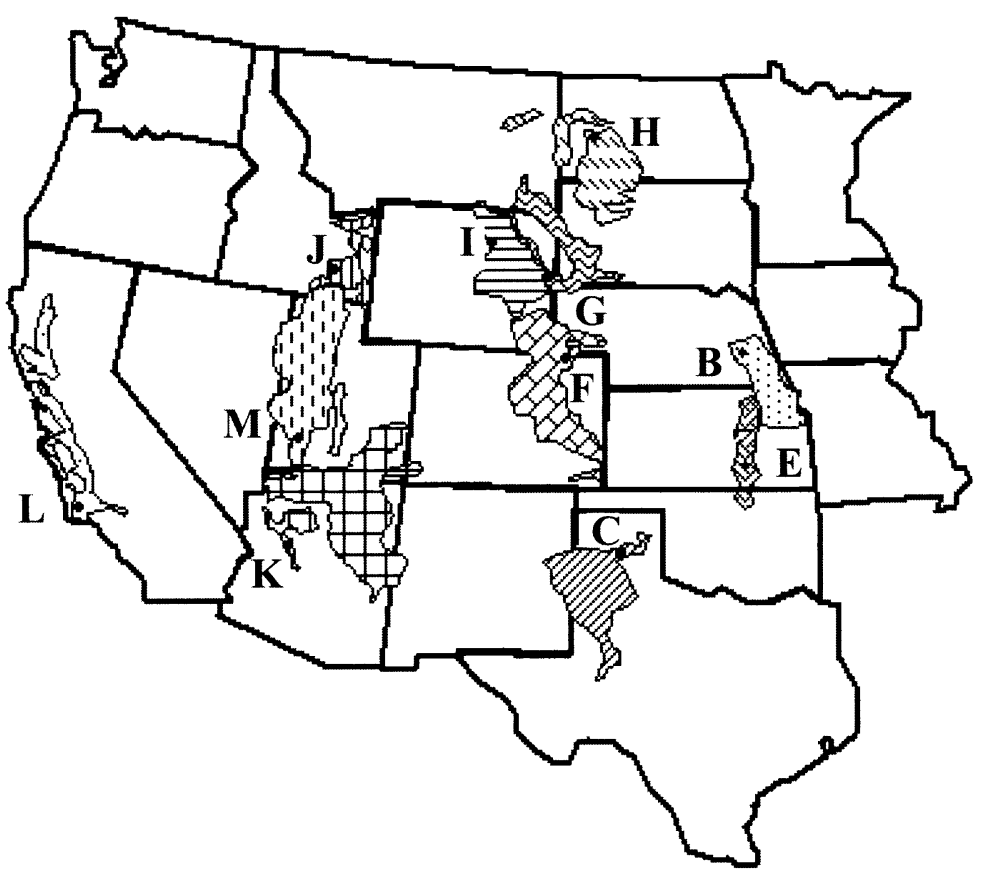

\begin{tabular}{|c|c|c|c|}
\hline Site & Location & MLRA & Lat./Long. \\
\hline B & Wahoo, NE & M106 & $41^{\circ} 3^{\prime} 58^{\prime \prime} \mathrm{N}, 9^{\circ} 53^{\prime} 18^{\prime \prime} \mathrm{W}$ \\
\hline $\mathbf{C}$ & Amarillo, TX & H77 & $35^{\circ} 16^{\prime} 30^{\prime \prime} \mathrm{N}, 102^{\circ} 16^{\prime} 42^{\prime \prime} \mathrm{W}$ \\
\hline $\mathbf{E}$ & Eureka, KS & H76 & $37^{\circ} 38^{\prime} 32^{\prime \prime} \mathrm{N}, 9^{\circ} \quad 10^{\prime} 48^{\prime \prime} \mathrm{W}$ \\
\hline$\nabla \mathbf{F}$ & Akron, CO & G67 & $40^{\circ} 22^{\prime} 26^{\prime \prime} \mathrm{N}, 103^{\circ} 7^{\prime} 42^{\prime \prime} \mathrm{W}$ \\
\hline 图 $\mathbf{G}$ & Newcastle, WY & G60A & $43^{\circ} 45^{\prime} 00^{\prime \prime} \mathrm{N}, 104^{\circ} 22^{\prime} 30^{\prime \prime} \mathrm{W}$ \\
\hline H & Killdeer, ND & F54 & $47^{\circ} 25^{\prime} 30^{\prime \prime} \mathrm{N}, 102^{\circ} 52^{\prime} 45^{\prime \prime} \mathrm{W}$ \\
\hline 目 I & Buffalo, WY & G58B & $44^{\circ} 20^{\prime} 32^{\prime \prime} \mathrm{N}, 106^{\circ} 31^{\prime} 38^{\prime \prime} \mathrm{W}$ \\
\hline 而 $\mathbf{J}$ & Blackfoot, ID & A13 & $43^{\circ} 3^{\prime} 6^{\prime \prime} \mathrm{N}, 111^{\circ} 46^{\prime} 40^{\prime \prime} \mathrm{W}$ \\
\hline 田 K & Prescott, AZ & D35 & $34^{\circ} 46^{\prime} 41^{\prime \prime} \mathrm{N}, 112^{\circ} 37^{\prime} 57^{\prime \prime} \mathrm{W}$ \\
\hline $\mathbf{L}$ & S.L. Obispo, CA & C15 & $35^{\circ} 19^{\prime} 46^{\prime \prime} \mathrm{N}, 120^{\circ} 41^{\prime} 49^{\prime \prime} \mathrm{W}$ \\
\hline 四 & Cedar City, UT & D28A & $37^{\circ} 43^{\prime} 35^{\prime \prime} \mathrm{N}, 113^{\circ} 18^{\prime} 4^{\prime \prime} \mathrm{W}$ \\
\hline
\end{tabular}

Fig. 1. Location of sampling sites within the 11 Major Land Resource Areas chosen for study. tion. Current rangeland models are based on the following assumptions: 1) algorithms developed from abundant cropland data should apply to rangelands (Foster and Lane 1987) where limited data has hampered rangeland modeling efforts, and 2) site characteristics of diverse rangeland types can be lumped or averaged together to develop 1 algorithm or equation to describe a process or relationship across the entire spectrum of rangeland types (Alberts et al. 1995). This universal approach simplifies the modeling process and broadens the practical application of the rangeland model. These simpler, more generalized models may be easier to use and require fewer resources. However, they may also be unresponsive to, or inaccurate in describing, variable interactions that affect management decisions. They may be unresponsive if the important fac- tors that govern rangeland hydrology on a specific site are not included in the generalized algorithm. They may be inaccurate for a specific site if the relationship or interaction between factors is poorly represented for that site by the generalized algorithm.

Are these assumptions, and modeling approaches based on the universal concept, appropriate for use on diverse rangeland types? This paper presents a comprehensive data set of vegetation, soils, hydrology, and erosion relationships for diverse western rangelands. This initial data summary is used to assess the validity of these assumptions and determine their credibility for rangelands in general.

\section{Data Set}

\section{Background}

Process-based models can improve our understanding of system dynamics, and enhance our capability to predict disturbance and management impacts on rangelands. However, these types of endeavors have been hampered by the lack of comprehensive, interdisciplinary data sets for model development and testing. To address this problem, the Agricultural Research Service and the Natural Resources Conservation Service (NRCS) formed the National Range Study Team (NRST) in 1990. This team collected critical interdisciplinary field data pertaining to hydrologic response for a variety of important rangeland soil-vegetation assemblages across the western United States. The data were collected to assist in the development of infiltration and erosion modeling components for WEPP (Water Erosion Prediction Project, Flanagan and Livingston 1995) and similar processbased models and thus further our understanding of the complex soil-vegetationhydrology interactions that are particular to rangeland ecosystems.

\section{Study Sites}

Eleven Major Land Resource Areas (MLRAs) in 10 different western states were evaluated (Fig. 1). Two to 3 contrasting vegetation states within each of these MLRAs were chosen for comparative study based on their relevance to the region and to management (Table 1 ). Contrasting vegetation states were identified as transition states within the Ecological Site/Range Cover Type, with different ecological status (USDA-NRCS 1997) and/or plant species composition. All study areas were located on native 
Table 1. Location and primary features of each study site.

\begin{tabular}{|c|c|c|c|c|c|}
\hline State & $\begin{array}{l}\text { Site } \\
\text { ID }\end{array}$ & MLRA, Cover type & $\begin{array}{l}\text { Ecological } \\
\text { status }\end{array}$ & Slope & Dominant species \\
\hline NE & $\mathrm{B} 1$ & $\begin{array}{l}\text { NE/KS Loess-Drift Hills } \\
\text { (106), Bluestem prairie }\end{array}$ & $(\%)$ & $(\%)$ & $\begin{array}{l}\text { 1-Kentucky bluegrass (Poa pratensis L.) } \\
\text { 2-Dandelion (Taraxacum officinale G.H. Weber ex Wiggers) } \\
\text { 3-Alsike clover (Trifolium hybridum L.) }\end{array}$ \\
\hline NE & B2 & $\begin{array}{l}\text { NE/KS Loess-Drift Hills } \\
\text { (106), Bluestem prairie }\end{array}$ & 37 & 11 & $\begin{array}{l}\text { 1-Primrose (Primula spp.) } \\
\text { 2-Porcupinegrass (Stipa spartea (Trin.) Barkworth) } \\
\text { 3-Big bluestem (Andropogon gerardii Vitman) }\end{array}$ \\
\hline $\mathrm{TX}$ & $\mathrm{C} 1$ & $\begin{array}{l}\text { Southern High Plains (77), } \\
\text { Blue grama-buffalograss 2- }\end{array}$ & 72 & 3 & $\begin{array}{l}\text { 1-Blue grama (Bouteloua gracilis (Willd. Ex Kunth) Lag. Ex Griffiths) } \\
\text { 2-Buffalograss (Buchloe dactyloides (Nutt.) Engelm.) } \\
\text { 3-Prickly pear cactus (Opuntia polycantha Haw.) }\end{array}$ \\
\hline $\mathrm{TX}$ & $\mathrm{C} 2$ & $\begin{array}{l}\text { Southern High Plains ( } 77 \text { ), } \\
\text { Blue grama-buffalograss }\end{array}$ & 62 & 2 & $\begin{array}{l}\text { 1-Blue grama (Bouteloua gracilis (Willd. Ex Kunth) Lag. Ex Griffiths) } \\
\text { 2-Buffalograss (Buchloe dactyloides (Nutt.) Engelm.) } \\
\text { 3-Prickly pear cactus (Opuntia polycantha Haw.) }\end{array}$ \\
\hline KS & E1 & $\begin{array}{l}\text { Bluestem Hills ( } 76) \\
\text { Bluestem prairie }\end{array}$ & 7 & 5 & $\begin{array}{l}\text { 1-Annual broomweed (Gutierrezia dracunculoides (Pursh) Britt. \& Rusby) } \\
\text { 2- Missouri goldenrod (Solidago missouriensis Nutt.) } \\
\text { 3-Tall dropseed (Sporobolus asper (Poir.) Merr.) }\end{array}$ \\
\hline KS & E3 & $\begin{array}{l}\text { Bluestem Hills (76), } \\
\text { Bluestem prairie }\end{array}$ & 45 & 3 & $\begin{array}{l}\text { 1-Buffalograss (Buchloe dactyloides (Nutt.) Engelm.) } \\
\text { 2-Sideoats grama (Bouteloua curtipendula (Michx.) Torr.) } \\
\text { 3- Little bluestem (Schizachyrium scoparium (Michx.) Nash) }\end{array}$ \\
\hline $\mathrm{CO}$ & $\mathrm{F} 1$ & $\begin{array}{l}\text { Central High Plains (67), } \\
\text { Grama-Buffalograss }\end{array}$ & 76 & 7 & $\begin{array}{l}\text { 1-Blue grama (Bouteloua gracilis (Willd. Ex Kunth) Lag. Ex Griffiths) } \\
\text { 2-Western wheatgrass (Pascopyrum smithii (Rydb.) A. Love) } \\
\text { 3-Buffalograss (Buchloe dactyloides (Nutt.) Engelm.) }\end{array}$ \\
\hline $\mathrm{CO}$ & $\mathrm{F} 2$ & $\begin{array}{l}\text { Central High Plains (67), } \\
\text { Grama-Buffalograss }\end{array}$ & 44 & 8 & $\begin{array}{l}\text { 1-Blue grama (Bouteloua gracilis (Willd. Ex Kunth) Lag. Ex Griffiths) } \\
\text { 2-Sun sedge (Carex inops Baily spp. Heliophila (Mackenzie) Crins) } \\
\text { 3-Bottlebrush squirreltail (Elymus elymoides (Raf.) Swezey) }\end{array}$ \\
\hline $\mathrm{CO}$ & F3 & $\begin{array}{l}\text { Central High Plains (67), } \\
\text { Grama-Buffalograss }\end{array}$ & 45 & 7 & $\begin{array}{l}\text { 1-Buffalograss (Buchloe dactyloides (Nutt.) Engelm.) } \\
2 \text { Blue grama (Bouteloua gracilis (Willd. Ex Kunth) Lag. Ex Griffiths) } \\
\text { 3-Prickly pear cactus (Opuntia polycantha Haw.) }\end{array}$ \\
\hline ND & $\mathrm{H} 1$ & $\begin{array}{l}\text { Rolling Soft Shale Plain } \\
\text { (54), Prairie Sandreed- } \\
\text { Needlegrass }\end{array}$ & 60 & 12 & $\begin{array}{l}\text { 1-Needle-and-Thread (Hesperostipa comata (Trin. \& Rupr.) Barkworth) } \\
\text { 2-Prairie sandreed (Calamovilfa longifolia (Hook.) Scribn.) } \\
\text { 3-Sedge (Carex spp.) }\end{array}$ \\
\hline ND & $\mathrm{H} 2$ & $\begin{array}{l}\text { Rolling Soft Shale Plain } \\
\text { (54), Prairie Sandreed- } \\
\text { Needlegrass }\end{array}$ & 43 & 11 & $\begin{array}{l}\text { 1-Clubmoss (Lycopodium dendroideum Michx.) } \\
\text { 2-Sedge (Carex spp.) } \\
\text { 3-Crocus (Crocus L.) }\end{array}$ \\
\hline ND & $\mathrm{H} 3$ & $\begin{array}{l}\text { Rolling Soft Shale Plain } \\
\text { (54), Prairie Sandreed- } \\
\text { Needlegrass }\end{array}$ & 52 & 11 & $\begin{array}{l}\text { 1-Sedge (Carex spp.) } \\
\text { 2-Blue grama (Bouteloua gracilis (Willd. Ex Kunth) Lag. Ex Griffiths) } \\
\text { 3-Clubmoss (Lycopodium dendroideum Michx.) }\end{array}$ \\
\hline WY & I1 & $\begin{array}{l}\text { N. Rolling high Plains }(58 \mathrm{~B}) \text {, } \\
\text { Sagebrush-Grass Wheatgrass } \\
\text { Grama-Needlegrass }\end{array}$ & 33 & 10 & $\begin{array}{l}\text { 1-Big sagebrush (Artemisia tridentata Nutt. ssp wyomingensis Beetle \& Young) } \\
\text { 2-Prairie junegrass (Koleria macrantha (Ledeb.) J.A. Schultes) } \\
\text { 3-Western wheatgrass (Pascopyrum smithii (Rydb.) A. Love) }\end{array}$ \\
\hline WY & I2 & $\begin{array}{l}\text { N. Rolling high Plains }(58 \mathrm{~B}) \text {, } \\
\text { Sagebrush-Grass Wheatgrass- } \\
\text { Grama-Needlegrass }\end{array}$ & 40 & 7 & $\begin{array}{l}\text { 1-Western wheatgrass (Pascopyrum smithii (Rydb.) A. Love) } \\
\text { 2-Bluebunch wheatgrass (Pseudoroegneria spicata (Pursh) A. Love) } \\
\text { 3-Green needlegrass (Stipa viridula (Trin.) Barkworth) }\end{array}$ \\
\hline ID & $\mathrm{J} 1$ & $\begin{array}{l}\text { Eastern Idaho Plateau (13), } \\
\text { Mountain Big Sagebrush }\end{array}$ & 15 & 7 & $\begin{array}{l}\text { 1-Big sagebrush (Artemisia tridentata Nutt. ssp. Vaseyana (Rydb.) Boivin) } \\
\text { 2-Letterman's needlegrass (Achnatherum lettermanii (Vasey) Barkworth) } \\
\text { 3-Sandberg bluegrass (Poa secunda J. Presl) }\end{array}$ \\
\hline ID & $\mathrm{J} 2$ & $\begin{array}{l}\text { Eastern Idaho Plateau (13), } \\
\text { Mountain Big Sagebrush }\end{array}$ & 22 & 9 & $\begin{array}{l}\text { 1- Letterman's needlegrass (Achnatherum lettermanii (Vasey) Barkworth) } \\
\text { 2-Sandberg bluegrass (Poa secunda J. Presl) } \\
\text { 3-Prairie junegrass (Koleria macrantha (Ledeb.) J.A. Schultes) }\end{array}$ \\
\hline
\end{tabular}


Table 1. Continued.

\begin{tabular}{|c|c|c|c|c|c|}
\hline State & $\begin{array}{l}\text { Site } \\
\text { ID }\end{array}$ & MLRA, Cover type & $\begin{array}{l}\text { Ecological } \\
\text { status }\end{array}$ & Slope & Dominant species \\
\hline $\mathrm{AZ}$ & K1 & $\begin{array}{l}\mathrm{CO} \text { and Green River Plateaus } \\
(35) \text {, Grama-galleta }\end{array}$ & $\begin{array}{c}(\%) \\
54\end{array}$ & $\begin{array}{c}(\%) \\
5\end{array}$ & $\begin{array}{l}\text { (Descending order of \% composition by weight) } \\
\text { 1-Blue grama (Bouteloua gracilis (Willd. Ex Kunth) Lag. Ex Griffiths) } \\
\text { 2-Goldenweed (Haplopappus spp.) } \\
\text { 3-Ring muhly (Muhlenbergia torreyi (Kunth) A.S. Hithc. ex Bush) }\end{array}$ \\
\hline $\mathrm{AZ}$ & $\mathrm{K} 2$ & $\begin{array}{l}\mathrm{CO} \text { and Green River Plateaus } \\
\text { (35), Grama-galleta }\end{array}$ & 36 & 4 & $\begin{array}{l}\text { 1-Rubber rabbitbrush (Ericameria nauseosa (Pallas ex Pursh) Nesom \& Baird) } \\
\text { 2-Blue grama (Bouteloua gracilis (Willd. Ex Kunth) Lag. Ex Griffiths) } \\
\text { 3-Threeawn (Aristida ssp.) }\end{array}$ \\
\hline CA & L1 & $\begin{array}{l}\text { Central CA Coast Range (15), } \\
\text { Valley grassland }\end{array}$ & --- & 11 & $\begin{array}{l}\text { 1-Creatanweed (Hedypnois cretica (L.) Dum.-Cours.) } \\
\text { 2-Ryegrass (Lolium spp.) } \\
\text { 3-Burclover (Medicago polymorpha (L.) Beauv.) }\end{array}$ \\
\hline CA & L2 & $\begin{array}{l}\text { Central CA Coast Range (15), } \\
\text { Valley grassland }\end{array}$ & -- & 12 & $\begin{array}{l}\text { 1-Ryegrass (Lolium spp.) } \\
\text { 2-Purple falsebrome (Brachypodium distachyon (L.) Beauv.) } \\
\text { 3-Slender oat (Avena barbata Pott ex Link) }\end{array}$ \\
\hline UT & M1 & $\begin{array}{l}\text { Great Salt Lake Area ( } 28 \mathrm{~A}) \text {, } \\
\text { Wyoming big sagebrush }\end{array}$ & 35 & 3 & $\begin{array}{l}\text { 1-Big sagebrush (Artemisia tridentata Nutt. ssp wyomingensis Beetle \& Young) } \\
\text { 2-Bottlebrush squirreltail (Elymus elymoides (Raf.) Swezey) } \\
\text { 3-James' Galleta (Hilaria jamesii Torr.) }\end{array}$ \\
\hline UT & M2 & $\begin{array}{l}\text { Great Salt Lake Area ( } 28 \mathrm{~A}) \text {, } \\
\text { Wyoming big sagebrush }\end{array}$ & 24 & 3 & $\begin{array}{l}\text { 1- James' Galleta (Hilaria jamesii Torr.) } \\
\text { 2-Arrowfeather threeawn (Aristida purpurascens Poir.) } \\
\text { 3- Big sagebrush (Artemisia tridentata Nutt. ssp wyomingensis Beetle \& Young) }\end{array}$ \\
\hline
\end{tabular}

rangeland (no history of cultivation). Different vegetation states within a MLRA had similar soils and Potential Natural Communities (PNC); differences were assumed to be the result of past and current management. Site selection for each vegetation state within a MLRA was based on the benchmark site concept (Franks et al. 1993) to assure that representative sites integrated the important features of plant community, soils, and management history. Within each representative site, 6 sampling plots with similar slope were randomly selected. A complete description and history of study sites can be found in Franks et al. (1998).

\section{Methods}

Rainfall simulation using a rotating boom (Swanson 1965) was conducted simultaneously on two, $3.1 \times 10.7 \mathrm{~m}$ runoff plots. Detailed description of the rainfall simulation techniques used are presented by Simanton et al. $(1987,1991)$ and Franks et al. (1998). All 6 runoff plots per site were on undisturbed (not disturbed by farm implement) vegetation and soils. For each of the plots, 2 different rainfall simulation runs were conducted. The first run was conducted on the initial antecedent soil moisture with a target rainfall rate of $63.5 \mathrm{~mm} \mathrm{hr}^{-1}$ and lasted approximately 1 hour or until steady-state runoff occurred. This was termed the "dry" run. A "wet" run was conducted 24 hours later, utilizing the same target rainfall intensity and lasted until an equilibrium runoff rate was achieved. Runoff was measured continuously using small drop-box weirs equipped with a pressure transducer bub- bler gauge. Due to the differences in rainfall application rates typical of rotating boom simulators, actual water application rates and quantities were measured on each plot during the simulation run. Infiltration was calculated as the difference between measured rainfall and measured runoff over each 1 to 2 minute time interval throughout the simulation. Terminal infiltration rate was calculated as the difference between final rainfall intensity and final runoff rate. Sediment samples were collected in bottles at 1 to 2 minute intervals.

Because of the differences between sites in total rainfall application, weighted variables were calculated, in addition to cumulative totals, so that comparison of runoff and sediment could be made on sites with varying amount/duration of rainfall. These normalized variables included runoff/rainfall ratio $\left(\mathrm{mm} \mathrm{mm}^{-1}\right)$, which is the total runoff divided by total rainfall, and sediment/runoff ratio $\left(\mathrm{kg} \mathrm{ha}^{-1} \mathrm{~mm}^{-1}\right)$, which is the total sediment divided by total runoff.

Five soil pedons from each study site were characterized and sent to the NRCS National Soil Survey Center for analysis. One pedon was selected as representative of the site's soil phase and the others represented the typical range of soil surface characteristics. In addition, 6 to 12 soil bulk density samples were taken outside each plot using either the compliant cavity or the balloon technique (Blake and Hartge 1986). Samples were taken prior to the dry run and after the wet run at 2 different depths $(0-2.5 \mathrm{~cm}$, and $2.5-10 \mathrm{~cm})$ below the soil surface. Gravimetric soil moisture samples associated with each plot were collected 30 minutes prior to the dry run, 30 minutes prior to the wet run, and 30 minutes after the wet run. Three samples were collected from $0-5 \mathrm{~cm}$ depths, and 3 were collected from $5-20 \mathrm{~cm}$ depths (or to the bottom of the wetting front if less than $20 \mathrm{~cm}$ ). Selected average soil characteristics for each vegetation state within a MLRA are presented in Table 2.

Vegetation canopy and ground cover were evaluated using a point frame (Mueller-Dombois and Ellenberg 1974) with 10 systematic lines of 49 points per runoff plot. First hits were used to determine canopy cover (individual species recorded and standing dead) and ground hits were used to determine ground cover. Random roughness was measured at each pinpoint by determining pin height above/below an arbitrary line. The standard deviation of each of the 10 lines was then averaged for the composite random roughness value. In addition, the standing live and dead biomass $\left(\mathrm{kg} \mathrm{ha}^{-1}\right)$ of individual species/functional groups, and of litter and other surface residue were also measured for each plot by clipping/harvesting after the rainfall simulation, oven drying, and weighing. Root biomass for the surface 0 $10 \mathrm{~cm}$ of soil was estimated by wet sieving soil cores and drying and weighing remaining roots. Selected average vegetation characteristics for vegetation states within each MLRA are presented in Table 3.

All data were tested for normality, skewness and kurtosis. Normalized distributions were obtained for total sediment, runoff/rainfall ratio and sediment/runoff ratio by using a $\log _{10}$ transformation. An 
Table 2. Mean soil characteristics of each study site.

\begin{tabular}{|c|c|c|c|c|c|c|c|c|c|c|c|}
\hline State & $\begin{array}{l}\text { Site } \\
\text { ID }\end{array}$ & $\begin{array}{l}\text { Soil series, } \\
\text { Surface texture }\end{array}$ & $\begin{array}{l}\text { Surface } \\
\text { Rough- } \\
\text { ness }\end{array}$ & $\begin{array}{c}P_{b} \\
\text { Dry } \\
0-10 \mathrm{~cm}\end{array}$ & $\begin{array}{c}P_{b} \\
\text { Wet } \\
0-10 \mathrm{~cm}\end{array}$ & $\begin{array}{c}\text { Antec. } \\
\text { SM }^{1} \\
\text { Dry } \\
\text { Run }\end{array}$ & $\begin{array}{c}\text { Antec. } \\
\text { SM }^{1} \\
\text { Wet } \\
\text { Run }\end{array}$ & $\begin{array}{l}\text { Clay } \\
0-8 \\
\mathrm{~cm}\end{array}$ & $\begin{array}{l}\text { Sand } \\
0-8 \\
\mathrm{~cm}\end{array}$ & $\begin{array}{l}\text { Organic } \\
\text { Carbon } \\
0-8 \mathrm{~cm}\end{array}$ & $\begin{array}{l}\text { Aggregate } \\
\text { Stability }\end{array}$ \\
\hline & & & (m) & $\left(\mathrm{g} \mathrm{cm}^{-3}\right)$ & $\left(\mathrm{g} \mathrm{cm}^{-3}\right)$ & $(\%)$ & $(\%)$ & $(\%)$ & $(\%)$ & $(\%)$ & $(\%)$ \\
\hline \multirow[t]{2}{*}{ NE } & B1 & Burchard, loam & .0099 & 1.40 & 1.17 & 24.2 & 30.1 & 32.6 & 27.3 & 6.22 & 67.0 \\
\hline & B2 & Burchard, loam & .0101 & 1.19 & 1.00 & 19.9 & 25.7 & 27.9 & 35.9 & 3.95 & 81.2 \\
\hline \multirow[t]{2}{*}{$\mathrm{TX}$} & $\mathrm{C} 1$ & Olton, loam & .0081 & 1.14 & 1.14 & 7.1 & 23.1 & 22.8 & 32.3 & 2.39 & 78.2 \\
\hline & $\mathrm{C} 2$ & Olton, loam & .0072 & 1.31 & 1.29 & 10.9 & 20.8 & 23.1 & 35.9 & 2.21 & 54.0 \\
\hline \multirow[t]{3}{*}{$\mathrm{KS}$} & E1 & Martin, silty clay loam & .0116 & 1.10 & 0.97 & 18.6 & 30.1 & 44.3 & 4.8 & 4.23 & 86.0 \\
\hline & E2 & Martin, silty clay loam & .0092 & 1.04 & 1.12 & 34.2 & 34.0 & 40.6 & 11.1 & 4.36 & 60.8 \\
\hline & E3 & Martin, silty clay loam & .0088 & 1.01 & 1.01 & 18.7 & 33.6 & 43.4 & 2.7 & 4.34 & 56.8 \\
\hline \multirow[t]{3}{*}{$\mathrm{CO}$} & F1 & Stoneham, loam & .0098 & 1.10 & 1.07 & 5.6 & 21.7 & 21.7 & 51.9 & 1.45 & 57.6 \\
\hline & $\mathrm{F} 2$ & Stoneham, fine sandy loam & .0087 & 1.34 & 1.34 & 4.2 & 15.7 & 13.3 & 55.9 & 1.75 & 59.7 \\
\hline & F3 & Stoneham, loam & .0125 & 1.22 & 1.16 & 13.5 & 19.2 & 18.0 & 61.9 & 1.59 & 53.0 \\
\hline \multirow[t]{3}{*}{ WY } & G1 & Kishona, v. fine sandy loam & .0165 & 1.15 & 1.26 & 3.2 & 14.6 & 11.4 & 58.1 & 1.29 & 34.0 \\
\hline & $\mathrm{G} 2$ & Kishona, clay loam & .0192 & 1.21 & 1.14 & 6.6 & 15.5 & 23.7 & 47.1 & 1.76 & 41.8 \\
\hline & G3 & Kishona, $v$. fine sandy loam & .0207 & 1.11 & 1.17 & 7.1 & 15.7 & 13.6 & 60.1 & 1.30 & 11.0 \\
\hline \multirow[t]{3}{*}{ ND } & $\mathrm{H} 1$ & Parshall, sandy loam & .0101 & 1.25 & 1.16 & 6.5 & 16.3 & 15.3 & 61.3 & 2.70 & 83.8 \\
\hline & $\mathrm{H} 2$ & Parshall, fine sandy loam & .0113 & 1.03 & 1.09 & 19.9 & 19.4 & 14.4 & 63.8 & 3.72 & 85.3 \\
\hline & $\mathrm{H} 3$ & Parshall, sandy loam & .0097 & 1.2 & 1.31 & 15.1 & 19.6 & 11.3 & 70.3 & 3.13 & 74.0 \\
\hline \multirow[t]{2}{*}{ WY } & I1 & Forkwood, silt loam & .0269 & 1.11 & 1.02 & 15.9 & 23.1 & 23.6 & 34.9 & 2.21 & 17.3 \\
\hline & $\mathrm{I} 2$ & Forkwood, loam & .0156 & 1.17 & 1.10 & 16.6 & 24.0 & 29.5 & 37.9 & 1.52 & 30.0 \\
\hline \multirow[t]{2}{*}{ ID } & J1 & Robin, silt loam & .0308 & 0.95 & 0.82 & 8.5 & 25.2 & 17.4 & 16.3 & 5.60 & 46.8 \\
\hline & $\mathrm{J} 2$ & Robin, silt loam & .0259 & 0.93 & 0.81 & 7.8 & 27.5 & 17.4 & 14.8 & 8.06 & 67.2 \\
\hline \multirow[t]{2}{*}{$\mathrm{AZ}$} & $\mathrm{K} 1$ & Lonti, sandy loam & .0152 & 1.36 & 1.34 & 8.0 & 17.2 & 10.3 & 53.0 & 1.26 & 22.2 \\
\hline & $\mathrm{K} 2$ & Lonti, sandy loam & .0165 & 1.14 & 1.16 & 6.7 & 16.5 & 6.6 & 56.4 & 0.72 & 9.4 \\
\hline \multirow[t]{2}{*}{$\mathrm{CA}$} & $\mathrm{L} 1$ & Diablo, clay loam & .0162 & 1.39 & 1.29 & 14.6 & 24.9 & 37.9 & 32.7 & 1.76 & 69.3 \\
\hline & $\mathrm{L} 2$ & Diablo, silty clay & .0176 & 1.35 & 1.29 & 12.3 & 22.6 & 42.8 & 15.4 & 2.39 & 78.8 \\
\hline \multirow[t]{2}{*}{ UT } & M1 & Taylors Flat, sandy loam & .0193 & 0.99 & 1.20 & 11.1 & 18.7 & 10.7 & 67.3 & 0.60 & -.- \\
\hline & M2 & Taylors Flat, sandy loam & .0179 & 1.45 & 1.29 & 8.6 & 16.3 & 11.1 & 65.7 & 1.17 & 4.5 \\
\hline
\end{tabular}

${ }^{1}$ Gravimetric antecedent soil moisture

Table 3. Mean vegetative characteristics of each study site.

\begin{tabular}{|c|c|c|c|c|c|c|c|c|c|c|c|c|}
\hline State & $\begin{array}{l}\text { Site } \\
\text { ID }\end{array}$ & $\begin{array}{l}\text { Grass } \\
\text { Cover }\end{array}$ & $\begin{array}{c}\text { Grass \& } \\
\text { Forb } \\
\text { Cover }\end{array}$ & $\begin{array}{c}\text { Shrub \& } \\
\text { Cactus } \\
\text { Cover }\end{array}$ & $\begin{array}{c}\text { Bare } \\
\text { Ground }\end{array}$ & $\begin{array}{l}\text { Litter } \\
\text { Cover }\end{array}$ & $\begin{array}{c}\text { Annual } \\
\text { Grass } \\
\text { Biomass }\end{array}$ & $\begin{array}{c}\text { Perennial } \\
\text { Grass } \\
\text { Biomass }\end{array}$ & $\begin{array}{l}\text { Shrub } \\
\text { Biomass }\end{array}$ & $\begin{array}{c}\text { Litter } \\
\text { Biomass }\end{array}$ & $\begin{array}{c}\text { Total } \\
\text { Standing } \\
\text { Biomass }\end{array}$ & $\begin{array}{c}\text { Root } \\
\text { Biomass }\end{array}$ \\
\hline & & $(\%)$ & $(\%)$ & $(\%)$ & $(\%)$ & $(\%)$ & $\left(\mathrm{kg} \mathrm{ha}^{-1}\right)$ & $\left(\mathrm{kg} \mathrm{ha}^{-1}\right)$ & $\left(\mathrm{kg} \mathrm{ha}^{-1}\right)$ & $\left(\mathrm{kg} \mathrm{ha}^{-1}\right)$ & $\left(\mathrm{kg} \mathrm{ha}^{-1}\right)$ & $\left(\mathrm{kg} \mathrm{ha}^{-1}\right)$ \\
\hline \multirow[t]{2}{*}{ NE } & B1 & 9.4 & 27.3 & 0 & 20.4 & 72.9 & 12 & 714 & 0 & 575 & 1100 & 4798 \\
\hline & $\mathrm{B} 2$ & 10.2 & 17.4 & 5.2 & 11.6 & 86.0 & 167 & 1849 & 207 & 763 & 3443 & 1653 \\
\hline \multirow[t]{2}{*}{$\mathrm{TX}$} & $\mathrm{C} 1$ & 9.8 & 10.9 & 0.5 & 3.0 & 83.2 & 0 & 1111 & 101 & 2383 & 1331 & 4695 \\
\hline & $\mathrm{C} 2$ & 8.9 & 8.9 & $\mathrm{~T}^{1}$ & 13.3 & 83.7 & 0 & 509 & 2 & 1694 & 510 & 5532 \\
\hline \multirow[t]{3}{*}{$\mathrm{KS}$} & E1 & 21.8 & 54.5 & 0.2 & 27.4 & 70.5 & 128 & 269 & 0 & 1679 & 1732 & 2573 \\
\hline & $\mathrm{E} 2$ & 52.2 & 58.8 & $\mathrm{~T}$ & 23.6 & 74.3 & 0 & 1927 & 43 & 1310 & 2069 & 8593 \\
\hline & E3 & 32.2 & 37.2 & 0 & 41.8 & 55.2 & 5 & 404 & 15 & 387 & 508 & 6651 \\
\hline \multirow[t]{3}{*}{$\mathrm{CO}$} & F1 & 49.8 & 50.5 & 0 & 4.4 & 76.9 & 0 & 1124 & 0 & 2130 & 1126 & 4615 \\
\hline & $\mathrm{F} 2$ & 42.0 & 42.2 & 0.1 & 14.4 & 57.3 & 0 & 830 & 0 & 1753 & 831 & 6357 \\
\hline & F3 & 27.2 & 27.4 & 0.4 & 17.9 & 68.6 & 0 & 411 & 15 & 1789 & 427 & 11615 \\
\hline \multirow[t]{3}{*}{ WY } & G1 & 6.0 & 7.0 & 3.0 & 23.2 & 40.0 & 27 & 735 & 39 & 423 & 802 & 10239 \\
\hline & $\mathrm{G} 2$ & 53.9 & 54.8 & 0.7 & 18.9 & 77.4 & 763 & 1104 & 7 & 1047 & 1879 & 4021 \\
\hline & G3 & 27.6 & 30.1 & 0.5 & 53.1 & 32.1 & 0 & 722 & 4 & 174 & 766 & 8144 \\
\hline \multirow[t]{3}{*}{ ND } & $\mathrm{Hl}$ & 48.3 & 50.7 & 3.0 & 3.5 & 92.0 & 0 & 700 & 4 & 1534 & 810 & 6761 \\
\hline & $\mathrm{H} 2$ & 46.9 & 63.8 & 0.5 & 3.8 & 64.1 & 0 & 692 & 5 & 655 & 1416 & 7482 \\
\hline & $\mathrm{H} 3$ & 59.6 & 67.3 & 1.1 & 11.6 & 69.4 & 0 & 832 & 2 & 1139 & 1221 & 4089 \\
\hline \multirow[t]{2}{*}{ WY } & II & 19.4 & 21.0 & 28.3 & 38.4 & 55.6 & 0 & 425 & 494 & 1103 & 1730 & 3413 \\
\hline & $\mathrm{I} 2$ & 41.9 & 47.8 & $\mathrm{~T}$ & 36.5 & 54.9 & 29 & 872 & 0 & 796 & 2246 & 1881 \\
\hline \multirow[t]{2}{*}{ ID } & $\mathrm{J} 1$ & 29.7 & 30.6 & 38.9 & 10.1 & 84.6 & 0 & 297 & 11 & 6413 & 774 & 3344 \\
\hline & $\mathrm{J} 2$ & 78.6 & 82.7 & 3.3 & 7.4 & 84.4 & 0 & 1194 & 112 & 4428 & 1422 & 7362 \\
\hline \multirow[t]{2}{*}{$\mathrm{AZ}$} & $\mathrm{K} 1$ & 31.2 & 45.8 & 1.6 & 50.7 & 28.7 & 29 & 474 & 3 & 216 & 782 & 912 \\
\hline & $\mathrm{K} 2$ & 34.6 & 39.1 & 10.6 & 49.8 & 26.0 & . 3 & 566 & 481 & 735 & 1159 & 759 \\
\hline \multirow[t]{2}{*}{$\mathrm{CA}$} & $\mathrm{L} 1$ & 43.3 & 96.3 & 0 & 50.3 & 37.4 & 1171 & 0 & 16 & 177 & 2621 & 668 \\
\hline & $\mathrm{L} 2$ & 74.4 & 77.4 & 0 & 6.2 & 90.1 & 754 & 22 & 0 & 1730 & 817 & 883 \\
\hline \multirow[t]{2}{*}{ UT } & M1 & 3.0 & 5.1 & 20.7 & 59.6 & 29.5 & 0 & 45 & 405 & 4936 & 464 & 608 \\
\hline & M2 & 25.3 & 30.0 & 1.3 & 58.2 & 37.0 & 1 & 604 & 19 & 2717 & 3306 & 1490 \\
\hline
\end{tabular}


Table 4. Differences in cumulative infiltration $(\mathrm{mm})$, terminal infiltration rate $\left(\mathrm{mm} \mathrm{hr}^{-1}\right)$, runoff/rainfall ratio $\left(\mathrm{mm} \mathrm{mm}^{-1}\right)$, total sediment $\left(\mathrm{kg} \mathrm{ha}^{-1}\right)$, and sediment/runoff ratio $\left(\mathrm{kg} \mathrm{ha}^{-1}\right)$ for each study site under dry antecedent soil moisture conditions. Means within a column and within a region/cover type followed by different letters are significantly different $(P<0.10)$.

\begin{tabular}{|c|c|c|c|c|c|c|}
\hline State & $\begin{array}{l}\text { Site } \\
\text { ID }\end{array}$ & $\begin{array}{l}\text { Cumulative } \\
\text { Infiltration }\end{array}$ & $\begin{array}{c}\text { Terminal } \\
\text { Infiltration } \\
\text { Rate } \\
\end{array}$ & $\begin{array}{c}\text { Runoff/ } \\
\text { Rainfall } \\
\text { Ratio }\end{array}$ & $\begin{array}{c}\text { Total } \\
\text { Sediment }\end{array}$ & $\begin{array}{c}\text { Sediment/ } \\
\text { Runoff } \\
\text { Ratio }\end{array}$ \\
\hline & & $(\mathrm{mm})$ & $\left(\mathrm{mm} \mathrm{hr}^{-1}\right)$ & $\left(\mathrm{mm} \mathrm{mm}^{-1}\right)$ & $\left(\mathrm{kg} \mathrm{ha}^{-1}\right)$ & $\left(\mathrm{kg} \mathrm{ha}^{-1} \mathrm{~mm}^{-1}\right)$ \\
\hline \multirow[t]{2}{*}{$\mathrm{NE}$} & B1 & $26.6 \mathrm{a}$ & $19.1 \mathrm{a}$ & $0.51 b$ & $234.9 b$ & $8.8 \mathrm{~b}$ \\
\hline & B2 & $51.3 b$ & $49.7 b^{1}$ & $0.00 \mathrm{a}$ & $1.3 \mathrm{a}$ & $1.9 \mathrm{a}$ \\
\hline \multirow[t]{2}{*}{$\mathrm{TX}$} & $\mathrm{Cl}$ & $46.9 b$ & $41.7 b$ & $0.13 \mathrm{a}$ & $68.7 \mathrm{a}$ & $9.8 \mathrm{a}$ \\
\hline & $\mathrm{C} 2$ & $31.2 \mathrm{a}$ & $14.8 \mathrm{a}$ & $0.44 b$ & $235.6 b$ & $10.0 \mathrm{a}$ \\
\hline \multirow[t]{3}{*}{$\mathrm{KS}$} & E1 & $57.4 \mathrm{a}$ & $54.5 \mathrm{a}^{1}$ & $0.00 \mathrm{a}$ & $1.4 \mathrm{a}$ & $12.1 \mathrm{a}$ \\
\hline & E2 & $58.1 \mathrm{a}$ & $58.1 \mathrm{a}^{1}$ & $0.00 \mathrm{a}$ & $0.3 \mathrm{a}$ & $2.8 \mathrm{a}$ \\
\hline & E3 & $55.4 \mathrm{a}$ & $52.2 \mathrm{a}$ & $0.04 b$ & $17.7 \mathrm{~b}$ & $7.4 \mathrm{a}$ \\
\hline \multirow[t]{3}{*}{$\mathrm{CO}$} & F1 & $42.4 \mathrm{a}$ & $32.8 \mathrm{a}$ & $0.28 \mathrm{a}$ & $121.7 \mathrm{a}$ & $8.3 \mathrm{a}$ \\
\hline & $\mathrm{F} 2$ & $38.6 a$ & $31.6 \mathrm{a}$ & $0.33 a$ & $119.9 \mathrm{a}$ & $6.6 \mathrm{a}$ \\
\hline & F3 & $35.8 \mathrm{a}$ & $37.8 \mathrm{a}$ & $0.36 \mathrm{a}$ & $488.5 b$ & $27.2 b$ \\
\hline \multirow[t]{3}{*}{ WY } & G1 & $46.9 b$ & $48.1 \mathrm{~b}$ & $0.04 a$ & $21.3 \mathrm{a}$ & $7.3 \mathrm{a}$ \\
\hline & G2 & $53.7 \mathrm{c}$ & $54.3 \mathrm{~b}$ & $0.05 a$ & $29.6 a$ & $12.7 \mathrm{a}$ \\
\hline & G3 & $39.6 \mathrm{a}$ & $32.5 a$ & $0.30 \mathrm{~b}$ & $293.1 b$ & $17.3 \mathrm{a}$ \\
\hline \multirow[t]{3}{*}{ ND } & H1 & $48.8 \mathrm{a}$ & $53.5 b$ & $0.09 a$ & $24.1 \mathrm{a}$ & $6.9 a$ \\
\hline & $\mathrm{H} 2$ & $50.4 \mathrm{a}$ & $53.0 \mathrm{ab}$ & $0.08 \mathrm{a}$ & $73.1 \mathrm{~b}$ & $17.4 \mathrm{~b}$ \\
\hline & $\mathrm{H} 3$ & $48.3 \mathrm{a}$ & $49.3 \mathrm{a}$ & $0.12 \mathrm{a}$ & $47.5 \mathrm{ab}$ & $6.9 a$ \\
\hline \multirow[t]{2}{*}{ WY } & I1 & $52.9 \mathrm{a}$ & $51.4 \mathrm{~b}$ & $0.05 a$ & $77.6 a$ & $17.5 \mathrm{a}$ \\
\hline & I2 & $47.1 \mathrm{a}$ & $42.5 \mathrm{a}$ & $0.14 \mathrm{a}$ & $113.5 \mathrm{a}$ & $10.2 \mathrm{a}$ \\
\hline \multirow[t]{2}{*}{ ID } & $\mathrm{J} 1$ & $50.0 \mathrm{a}$ & $49.3 a$ & $0.08 \mathrm{a}$ & $28.8 \mathrm{a}$ & $6.0 \mathrm{~b}$ \\
\hline & $\mathrm{J} 2$ & $48.7 \mathrm{a}$ & $47.8 \mathrm{a}$ & $0.11 \mathrm{a}$ & $18.0 \mathrm{a}$ & $3.5 \mathrm{a}$ \\
\hline \multirow[t]{2}{*}{$\mathrm{AZ}$} & $\mathrm{K} 1$ & $42.8 \mathrm{a}$ & $39.7 a$ & $0.23 a$ & $195.7 a$ & $11.5 \mathrm{a}$ \\
\hline & $\mathrm{K} 2$ & $38.0 \mathrm{a}$ & $33.6 \mathrm{a}$ & $0.31 \mathrm{a}$ & $158.6 \mathrm{a}$ & $9.5 a$ \\
\hline \multirow[t]{2}{*}{$\mathrm{CA}$} & L1 & $56.7 \mathrm{a}$ & $56.2 \mathrm{a}^{1}$ & $0.0 \mathrm{a}$ & $0.3 \mathrm{a}$ & $1.1 \mathrm{a}$ \\
\hline & L2 & $60.4 b$ & $50.0 \mathrm{a}$ & $0.02 \mathrm{a}$ & $6.7 a$ & $1.5 \mathrm{a}$ \\
\hline \multirow[t]{2}{*}{ UT } & M1 & $55.3 \mathrm{a}$ & $53.4 \mathrm{a}$ & $0.05 b$ & $49.2 b$ & $20.3 b$ \\
\hline & $\mathrm{M} 2$ & $56.9 \mathrm{a}$ & $55.4 \mathrm{a}$ & $0.01 \mathrm{a}$ & $7.8 \mathrm{a}$ & $9.3 \mathrm{a}$ \\
\hline
\end{tabular}

were also the same grassland regions that showed differences in texture and aggregate stability between the vegetation states. The tallgrass prairie in Kansas (E), the annual grassland in California (L), and the mixed-grass prairie in North Dakota $(\mathrm{H})$ showed only slight differences in cumulative infiltration, terminal infiltration rate, or total sediment among the different vegetation states studied. The shrub-steppe rangeland types exhibited few significant differences in cumulative infiltration or terminal infiltration rate. All sagebrush sites in Wyoming (I), Idaho (J), and Utah (M) tended to have greater sediment/runoff ratios for the sites without brush management, but this difference was only significant for Idaho (J) and Utah (M).

Differences in cumulative infiltration, terminal infiltration, and runoff/rainfall ratio between vegetation states were more apparent during the wet runs (Table 5). Only the annual grassland (L) and the Colorado shortgrass prairie $(\mathrm{F})$ did not show significant differences in cumulative infiltration and/or terminal infiltration rate between their respective vegetation states. Vegetation states within grassland sites differed in total sediment and/or sedi-

${ }^{1}$ Infiltration rate was greater than application rainfall rate.

Table 5. Differences in cumulative infiltration $(\mathrm{mm})$, terminal infiltration rate $\left(\mathrm{mm} \mathrm{hr}^{-1}\right)$, runoff/rainfall ratio $\left(\mathrm{mm} \mathrm{mm}^{-1}\right)$, total sediment $\left(\mathrm{kg} \mathrm{ha}^{-1}\right)$, and sediment/runoff ratio $\left(\mathrm{kg} \mathrm{ha}^{-1}\right)$ for each study site under wet antecedent soil moisture conditions. Means within a column and within a region/cover type followed by different letters are significantly different $(P<0.10)$.

analysis of variance was performed on vegetation states within each MLRA, and across MLRAs (with nesting), using SAS (SAS Institute 1999) General Linear Models. Treatment means were separated using the Student-Neuman-Kuels multiple range test with $\mathrm{P} \leq 0.10$. The degree of linear association of variables most related to infiltration and erosion were evaluated using SAS Correlation procedures (Pearson correlation matrix). Forward multiple regression analysis $(P \leq 0.15)$ identified variables that may estimate infiltration and erosion across MLRAs. Differences discussed in the text are statistically significant at the defined P-values unless indicated otherwise.

\section{Results}

\section{Vegetation States Within MLRA}

The vegetation states within the tallgrass prairie in Nebraska (B), the shortgrass prairie in Texas (C), and the mixed-grass prairie in Wyoming $(\mathrm{G})$ showed the greatest contrast in cumulative infiltration, terminal infiltration rate, runoff/rainfall ratio, and total sediment under dry conditions (Table 4). Vegetation states of these grasslands had very dramatic differences in vegetation composition (Table 1), and

\begin{tabular}{|c|c|c|c|c|c|c|}
\hline State & $\begin{array}{l}\text { Site } \\
\text { ID }\end{array}$ & $\begin{array}{l}\text { Cumulative } \\
\text { Infiltration }\end{array}$ & $\begin{array}{c}\text { Terminal } \\
\text { Infiltration } \\
\text { Rate }\end{array}$ & $\begin{array}{c}\text { Runoff/ } \\
\text { Rainfall } \\
\text { Ratio }\end{array}$ & $\begin{array}{c}\text { Total } \\
\text { Sediment }\end{array}$ & $\begin{array}{c}\text { Sediment/ } \\
\text { Runoff } \\
\text { Ratio } \\
\end{array}$ \\
\hline & & $(\mathrm{mm})$ & $\left(\mathrm{mm} \mathrm{hr}^{-1}\right)$ & $\left(\mathrm{mm} \mathrm{mm}^{-1}\right)$ & $\left(\mathrm{kg} \mathrm{ha}^{-1}\right)$ & $\left(\mathrm{kg} \mathrm{ha}^{-1} \mathrm{~mm}^{-1}\right)$ \\
\hline \multirow[t]{2}{*}{$\mathrm{NE}$} & B1 & $8.4 \mathrm{a}$ & $17.7 \mathrm{a}$ & $0.50 \mathrm{~b}$ & $29.6 \mathrm{a}$ & $3.6 \mathrm{a}$ \\
\hline & B2 & $25.1 \mathrm{~b}$ & $43.9 \mathrm{~b}$ & $0.07 a$ & $21.4 a$ & $11.9 \mathrm{~b}$ \\
\hline \multirow[t]{2}{*}{ TX } & $\mathrm{C} 1$ & $25.4 \mathrm{~b}$ & $40.5 b$ & $0.14 a$ & $48.3 \mathrm{a}$ & $11.2 \mathrm{~b}$ \\
\hline & $\mathrm{C} 2$ & $15.5 \mathrm{a}$ & $17.4 \mathrm{a}$ & $0.46 \mathrm{~b}$ & $78.4 a$ & $6.2 \mathrm{a}$ \\
\hline \multirow[t]{3}{*}{$\mathrm{KS}$} & E1 & $48.0 \mathrm{~b}$ & $50.6 \mathrm{~b}$ & $0.02 a$ & $17.2 \mathrm{a}$ & $10.6 \mathrm{~b}$ \\
\hline & E2 & $56.5 \mathrm{~b}$ & $53.3 b^{1}$ & $0.00 \mathrm{a}$ & $0.0 \mathrm{a}$ & $0.0 \mathrm{a}$ \\
\hline & E3 & $16.3 \mathrm{a}$ & $29.2 \mathrm{a}$ & $0.39 b$ & $92.7 \mathrm{~b}$ & $8.9 \mathrm{~b}$ \\
\hline \multirow[t]{3}{*}{$\mathrm{CO}$} & $\mathrm{F} 1$ & $19.2 \mathrm{a}$ & $18.6 \mathrm{a}$ & $0.49 b$ & $81.9 \mathrm{a}$ & $5.2 \mathrm{a}$ \\
\hline & F2 & $23.2 \mathrm{a}$ & $33.5 \mathrm{a}$ & $0.29 a$ & $88.8 \mathrm{a}$ & 9.7ab \\
\hline & F3 & $18.5 \mathrm{a}$ & $20.8 \mathrm{a}$ & $0.47 \mathrm{~b}$ & $195.8 \mathrm{~b}$ & $12.8 \mathrm{~b}$ \\
\hline \multirow{3}{*}{ WY } & G1 & $44.6 \mathrm{~b}$ & $40.7 \mathrm{~b}$ & $0.10 \mathrm{a}$ & $75.6 \mathrm{~b}$ & $19.6 \mathrm{a}$ \\
\hline & G2 & $37.4 b$ & $52.1 \mathrm{c}$ & $0.06 \mathrm{a}$ & $17.7 \mathrm{a}$ & $7.6 \mathrm{a}$ \\
\hline & G3 & $20.9 a$ & $24.6 \mathrm{a}$ & $0.38 \mathrm{~b}$ & $134.5 \mathrm{~b}$ & $10.7 \mathrm{a}$ \\
\hline \multirow[t]{3}{*}{ ND } & $\mathrm{H} 1$ & $51.7 \mathrm{~b}$ & $58.3 b^{1}$ & $0.00 \mathrm{a}$ & $0.6 \mathrm{a}$ & $5.4 \mathrm{a}$ \\
\hline & $\mathrm{H} 2$ & $31.1 \mathrm{a}$ & $36.0 \mathrm{a}$ & $0.18 \mathrm{~b}$ & $85.3 b$ & $10.2 a$ \\
\hline & $\mathrm{H} 3$ & $25.8 \mathrm{a}$ & $33.4 \mathrm{a}$ & $0.22 b$ & $67.8 \mathrm{~b}$ & $8.5 \mathrm{a}$ \\
\hline \multirow{2}{*}{ WY } & I1 & $21.5 \mathrm{a}$ & $42.6 \mathrm{~b}$ & $0.14 \mathrm{a}$ & $83.8 \mathrm{a}$ & $17.9 \mathrm{a}$ \\
\hline & I2 & $20.3 \mathrm{a}$ & $27.1 \mathrm{a}$ & $0.36 \mathrm{~b}$ & $113.1 \mathrm{a}$ & $9.1 \mathrm{a}$ \\
\hline \multirow[t]{2}{*}{ ID } & J1 & $40.6 \mathrm{~b}$ & $44.4 \mathrm{a}$ & $0.11 \mathrm{a}$ & $38.8 \mathrm{a}$ & 7.1a \\
\hline & $\mathrm{J} 2$ & $33.4 \mathrm{a}$ & $50.9 \mathrm{a}$ & $0.07 \mathrm{a}$ & $8.9 \mathrm{a}$ & $4.0 \mathrm{a}$ \\
\hline \multirow[t]{2}{*}{$\mathrm{AZ}$} & K1 & $17.7 \mathrm{a}$ & $27.1 \mathrm{a}$ & $0.36 \mathrm{a}$ & $75.3 a$ & $7.2 \mathrm{a}$ \\
\hline & K2 & $17.2 \mathrm{a}$ & $28.8 \mathrm{a}$ & $0.36 \mathrm{a}$ & $100.6 a$ & $10.0 \mathrm{a}$ \\
\hline \multirow[t]{2}{*}{$\mathrm{CA}$} & L1 & $35.9 a$ & $14.0 \mathrm{a}$ & $0.42 \mathrm{a}$ & $179.4 \mathrm{~b}$ & $6.8 \mathrm{~b}$ \\
\hline & L2 & $28.7 \mathrm{a}$ & $7.0 \mathrm{a}$ & $0.41 \mathrm{a}$ & $88.3 \mathrm{a}$ & $4.5 \mathrm{a}$ \\
\hline \multirow[t]{2}{*}{ UT } & M1 & $21.8 \mathrm{a}$ & $38.1 \mathrm{a}$ & $0.24 a$ & $110.9 \mathrm{a}$ & $19.4 \mathrm{a}$ \\
\hline & M2 & $58.8 \mathrm{~b}$ & $45.6 \mathrm{a}$ & $0.50 \mathrm{~b}$ & $163.0 \mathrm{a}$ & $17.0 \mathrm{a}$ \\
\hline
\end{tabular}

Infiltration rate was greater than application rainfall rate. 
Table 6. Correlation coefficients for various canopy cover, ground cover, above-ground biomass, root biomass, and soil characteristic variable classes for total (cumulative) infiltration, terminal infiltration rate, total (cumulative) sediment (log10), and sediment/runoff ratio (log 10) on mixed and tall grass study sites. Only the highest correlated variable in each class and run is presented (some sites had few correlated variables). Correlations were performed for each state/region/cover type for both the dry and wet runs.

\begin{tabular}{|c|c|c|c|c|c|c|c|c|c|c|c|}
\hline \multirow[t]{2}{*}{$\begin{array}{c}\text { State, Region, } \\
\text { Cover Type }\end{array}$} & \multirow[t]{2}{*}{$\begin{array}{c}\text { Hydrologic/Erosion } \\
\text { Parameter }\end{array}$} & \multicolumn{2}{|c|}{$\begin{array}{c}\text { Canopy Cover } \\
\text { Variables }^{1}\end{array}$} & \multicolumn{2}{|c|}{$\begin{array}{c}\text { Ground Cover } \\
\text { Variables }^{2}\end{array}$} & \multicolumn{2}{|c|}{$\begin{array}{c}\text { Above-ground } \\
\text { Biomass Variables }^{3}\end{array}$} & \multicolumn{2}{|c|}{$\begin{array}{c}\text { Root Biomass } \\
\text { Variables }^{4}\end{array}$} & \multicolumn{2}{|c|}{$\begin{array}{c}\text { Soil } \\
\text { Variables }^{5}\end{array}$} \\
\hline & & Dry & Wet & Dry & Wet & Dry & Wet & Dry & Wet & Dry & Wet \\
\hline \multirow{4}{*}{$\begin{array}{l}\text { Nebraska, } \\
\text { Tallgrass } \\
\text { prairie, } \\
\text { Bluestem } \\
\text { prairie }\end{array}$} & $\begin{array}{l}\text { 1. Total Infiltration } \\
(\mathrm{mm})\end{array}$ & $\begin{array}{c}-0.89 \\
\text { (forbs) }\end{array}$ & $\begin{array}{c}-0.95 \\
\text { (forbs) }\end{array}$ & $\begin{array}{c}-0.79 \\
\text { (basal) }\end{array}$ & $\begin{array}{c}-0.75 \\
\text { (basal) }\end{array}$ & $\begin{array}{c}0.85 \\
\text { (p grass) }\end{array}$ & $\begin{array}{c}0.94 \\
\text { (total) }\end{array}$ & & $\begin{array}{c}-0.72 \\
\text { (average) }\end{array}$ & $\begin{array}{c}0.87 \\
\text { (ag stab) }\end{array}$ & $\begin{array}{c}0.99 \\
\text { (ag. stab) }\end{array}$ \\
\hline & $\begin{array}{l}\text { 2. Terminal Infiltration } \\
\text { Rate }\left(\mathrm{mm} \mathrm{hr}^{-1}\right)\end{array}$ & $\begin{array}{c}-0.90 \\
\text { (forbs) }\end{array}$ & $\begin{array}{c}-0.83 \\
\text { (forbs) }\end{array}$ & $\begin{array}{c}0.73 \\
\text { (litter) }\end{array}$ & $\begin{array}{c}-0.56 \\
\text { (basal) }\end{array}$ & $\begin{array}{c}0.88 \\
\text { (p grass) }\end{array}$ & $\begin{array}{c}0.85 \\
\text { (total ) }\end{array}$ & $\begin{array}{c}-0.63 \\
\text { (average) }\end{array}$ & $\begin{array}{c}-0.71 \\
\text { (average) }\end{array}$ & $\begin{array}{c}-0.91 \\
\text { (sm surf) }\end{array}$ & $\begin{array}{c}-0.86 \\
\text { (sm surf) }\end{array}$ \\
\hline & $\begin{array}{l}\text { 3. Total Sediment } \\
\left(\mathrm{kg} \mathrm{ha}^{-1}\right)\end{array}$ & $\begin{array}{c}0.93 \\
\text { (forbs) }\end{array}$ & & $\begin{array}{c}0.81 \\
\text { (basal) }\end{array}$ & & $\begin{array}{c}-0.88 \\
\text { (p grass) }\end{array}$ & $\begin{array}{c}-0.55 \\
\text { (p grass) }\end{array}$ & $\begin{array}{c}0.76 \\
\text { (average) }\end{array}$ & & $\begin{array}{c}0.94 \\
\text { (sm surf) }\end{array}$ & $\begin{array}{c}0.64 \\
\text { (sm sub) }\end{array}$ \\
\hline & $\begin{array}{l}\text { 4. Sediment/Runoff } \\
\text { Ratio }\left(\mathrm{kg} \mathrm{ha}^{-1} \mathrm{~mm}^{-1}\right)\end{array}$ & $\begin{array}{c}0.73 \\
\text { (forbs) }\end{array}$ & $\begin{array}{c}-0.78 \\
\text { (forbs) }\end{array}$ & $\begin{array}{c}0.62 \\
\text { (basal) }\end{array}$ & $\begin{array}{c}-0.63 \\
\text { (basal) }\end{array}$ & $\begin{array}{c}-0.73 \\
\text { (a grass) }\end{array}$ & $\begin{array}{c}0.95 \\
\text { (shrubs) }\end{array}$ & $\begin{array}{c}0.74 \\
\text { (average) }\end{array}$ & $\begin{array}{c}-0.53 \\
\text { (average) }\end{array}$ & $\begin{array}{c}0.75 \\
\text { (sm surf) }\end{array}$ & $\begin{array}{c}0.86 \\
\text { (ag stab) }\end{array}$ \\
\hline \multirow{4}{*}{$\begin{array}{l}\text { Kansas, } \\
\text { Tallgrass } \\
\text { prairie, } \\
\text { Bluestem } \\
\text { prairie }\end{array}$} & $\begin{array}{l}\text { 1. Total Infiltration } \\
(\mathrm{mm})\end{array}$ & & & & $\begin{array}{c}0.71 \\
\text { (litter) }\end{array}$ & & $\begin{array}{c}0.66 \\
\text { (p grass) }\end{array}$ & & $\begin{array}{c}0.60 \\
\text { (surf.) }\end{array}$ & & $\begin{array}{c}-0.77 \\
\text { (clay ss) }\end{array}$ \\
\hline & $\begin{array}{l}\text { 2. Terminal Infiltration } \\
\text { Rate }\left(\mathrm{mm} \mathrm{hr}^{-1}\right)\end{array}$ & & & & $\begin{array}{c}0.80 \\
\text { (litter) }\end{array}$ & & $\begin{array}{c}0.74 \\
\text { (total) }\end{array}$ & & & & $\begin{array}{c}-0.78 \\
\text { (clay ss) }\end{array}$ \\
\hline & $\begin{array}{l}\text { 3. Total Sediment } \\
\left(\mathrm{kg} \mathrm{ha}^{-1}\right)\end{array}$ & & $\begin{array}{c}-0.61 \\
(\text { st dead })\end{array}$ & $\begin{array}{c}-0.78 \\
\text { (litter) }\end{array}$ & $\begin{array}{c}-0.74 \\
\text { (litter) }\end{array}$ & $\begin{array}{l}-0.57 \\
\text { (total) }\end{array}$ & $\begin{array}{l}-0.71 \\
(\text { total })\end{array}$ & & & $\begin{array}{c}0.70 \\
\text { (clay ss) }\end{array}$ & $\begin{array}{c}0.88 \\
\text { (clay ss) }\end{array}$ \\
\hline & $\begin{array}{l}\text { 4. Sediment/Runoff } \\
\text { Ratio }\left(\mathrm{kg} \mathrm{ha}^{-1} \mathrm{~mm}^{-1}\right)\end{array}$ & & $\begin{array}{l}-0.82 \\
\text { (grass) }\end{array}$ & $\begin{array}{c}0.59 \\
\text { (basal) }\end{array}$ & & $\begin{array}{c}-0.50 \\
\text { (litter) }\end{array}$ & $\begin{array}{c}-0.91 \\
\text { (p grass) }\end{array}$ & & $\begin{array}{c}-0.62 \\
\text { (average) }\end{array}$ & & $\begin{array}{c}0.94 \\
\text { (clay s) }\end{array}$ \\
\hline \multirow{4}{*}{$\begin{array}{l}\text { Wyoming, } \\
\text { Mixed grass } \\
\text { prairie, } \\
\text { Wheatgrass- } \\
\text { needlegrass }\end{array}$} & $\begin{array}{l}\text { 1. Total Infiltration } \\
(\mathrm{mm})\end{array}$ & & $\begin{array}{c}0.70 \\
\text { (cacti) }\end{array}$ & $\begin{array}{l}-0.62 \\
\text { (bare) }\end{array}$ & $\begin{array}{l}-0.65 \\
\text { (bare) }\end{array}$ & & & $\begin{array}{l}-0.69 \\
(\mathrm{sub})\end{array}$ & $\begin{array}{c}0.63 \\
\text { (int surf) }\end{array}$ & $\begin{array}{c}0.67 \\
\text { (ag stab) }\end{array}$ & $\begin{array}{c}0.68 \\
\text { (ag stab) }\end{array}$ \\
\hline & $\begin{array}{l}\text { 2. Terminal Infiltration } \\
\text { Rate }\left(\mathrm{mm} \mathrm{hr}^{-1}\right)\end{array}$ & $\begin{array}{c}-0.58 \\
\text { (forbs) }\end{array}$ & $\begin{array}{c}-0.65 \\
\text { (forbs) }\end{array}$ & $\begin{array}{l}-0.76 \\
\text { (bare) }\end{array}$ & $\begin{array}{l}-0.83 \\
\text { (bare) }\end{array}$ & & $\begin{array}{c}0.63 \\
\text { (total) }\end{array}$ & $\begin{array}{l}-0.64 \\
(\mathrm{sub})\end{array}$ & $\begin{array}{l}-0.61 \\
(\mathrm{sub})\end{array}$ & $\begin{array}{c}0.80 \\
(\text { ag stab) }\end{array}$ & $\begin{array}{c}0.84 \\
(\text { ag stab) }\end{array}$ \\
\hline & $\begin{array}{l}\text { 3. Total Sediment } \\
\left(\mathrm{kg} \mathrm{ha}^{-1}\right)\end{array}$ & $\begin{array}{c}0.60 \\
\text { (forbs) }\end{array}$ & $\begin{array}{c}0.52 \\
\text { (forbs) }\end{array}$ & $\begin{array}{l}0.75 \\
\text { (bare) }\end{array}$ & $\begin{array}{c}-0.62 \\
\text { (litter) }\end{array}$ & $\begin{array}{c}-0.78 \\
\text { (shrubs) }\end{array}$ & & & $\begin{array}{c}0.51 \\
\text { (average) }\end{array}$ & $\begin{array}{c}-0.62 \\
(\mathrm{ag} \mathrm{stab})\end{array}$ & $\begin{array}{c}-0.69 \\
(\mathrm{OC} \text { sub })\end{array}$ \\
\hline & $\begin{array}{l}\text { 4. Sediment/Runoff } \\
\text { Ratio }\left(\mathrm{kg} \mathrm{ha}^{-1} \mathrm{~mm}^{-1}\right)\end{array}$ & & & $\begin{array}{c}-0.62 \\
\text { (crypts) }\end{array}$ & & $\begin{array}{c}-0.77 \\
\text { (shrubs) }\end{array}$ & & & & & \\
\hline \multirow{4}{*}{$\begin{array}{l}\text { North Dakota, } \\
\text { Mixed grass } \\
\text { prairie, } \\
\text { Wheatgrass- } \\
\text { needlegrass }\end{array}$} & $\begin{array}{l}\text { 1. Total Infiltration } \\
(\mathrm{mm})\end{array}$ & & $\begin{array}{c}0.92 \\
\text { (st dead) }\end{array}$ & & $\begin{array}{c}0.83 \\
\text { (litter) }\end{array}$ & & $\begin{array}{c}-0.64 \\
\text { (total std) }\end{array}$ & & $\begin{array}{l}0.80 \\
\text { (sub) }\end{array}$ & & $\begin{array}{c}-0.75 \\
\text { (sm surf) }\end{array}$ \\
\hline & $\begin{array}{l}\text { 2. Terminal Infiltration } \\
\text { Rate }\left(\mathrm{mm} \mathrm{hr}^{-1}\right)\end{array}$ & & $\begin{array}{c}0.94 \\
\text { (st dead) }\end{array}$ & & $\begin{array}{c}0.91 \\
\text { (litter) }\end{array}$ & & $\begin{array}{c}-0.63 \\
\text { (total std) }\end{array}$ & $\begin{array}{c}0.52 \\
\text { (average) }\end{array}$ & $\begin{array}{l}0.82 \\
\text { (sub) }\end{array}$ & & $\begin{array}{c}-0.75 \\
\text { (sm surf) }\end{array}$ \\
\hline & $\begin{array}{l}\text { 3. Total Sediment } \\
\left(\mathrm{kg} \mathrm{ha}^{-1}\right)\end{array}$ & & $\begin{array}{c}-0.92 \\
\text { (st dead) }\end{array}$ & & $\begin{array}{l}-0.95 \\
\text { (litter) }\end{array}$ & & $\begin{array}{c}0.66 \\
\text { (total std) }\end{array}$ & & & $\begin{array}{c}0.52 \\
\text { (sm surf) }\end{array}$ & $\begin{array}{c}0.77 \\
\text { (sm surf) }\end{array}$ \\
\hline & $\begin{array}{l}\text { 4. Sediment/Runoff } \\
\text { Ratio }\left(\mathrm{kg} \mathrm{ha}^{-1} \mathrm{~mm}^{-1}\right)\end{array}$ & $\begin{array}{c}0.69 \\
\text { (forbs) }\end{array}$ & & $\begin{array}{c}0.54 \\
\text { (crypts) }\end{array}$ & $\begin{array}{c}-0.58 \\
\text { (litter) }\end{array}$ & $\begin{array}{c}0.53 \\
\text { (forbs) }\end{array}$ & & & & $\begin{array}{c}0.62 \\
\text { (sm surf) }\end{array}$ & \\
\hline
\end{tabular}

st dead=standing dead

${ }^{2}$ crypts $=$ cryptogams, bare=bareground

${ }^{3} \mathrm{p}$ grass=perennial grass, a grass=annual grass, total=total yield, tot $\mathrm{std}=$ total standing biomass

${ }^{4}$ average =average over soil sampled, surf=surface, sub=subsurface, int surf=interspace surface

${ }^{5} \mathrm{ag}$ stab=aggregate stability, sm surf=surface antecedent soil moisture, $\mathrm{sm}$ sub=subsurface antecedent soil moisture, clay $\mathrm{s}=$ surface clay content,

clay ss=subsurface clay content, $\mathrm{OC}$ sub=subsurface organic carbon

ment/runoff ratio, although the differences were not well correlated to runoff/rainfall ratios. There were no significant differences in sediment yield or sediment load between any of the vegetation states for shrub sites studied.

The vegetation and soil variables most correlated with infiltration and erosion are presented for tall and mixed grasslands (Table 6), shortgrass and annual grass- lands (Table 7), and shrub-steppe rangelands (Table 8). While there were a few variables that were common among some of the rangeland types, they varied widely in their significance. Litter, for example, was highly correlated with terminal infiltration rate $(\mathrm{R}=0.91)$ on the North Dakota mixed grass sites (H, Table 6), but only slightly correlated with terminal infiltration rate $(R=0.53)$ on the sagebrush sites of Utah (M, Table 8). Some variables were positively correlated on some sites, but negatively correlated on others. For example, shrub biomass on the Nebraska tallgrass sites (B) was positively correlated with sediment loading, but negatively correlated on the Wyoming mixed grass sites (G, Table 6). These correlations demonstrate the extreme diversity of rangelands located in different MLRAs with respect 


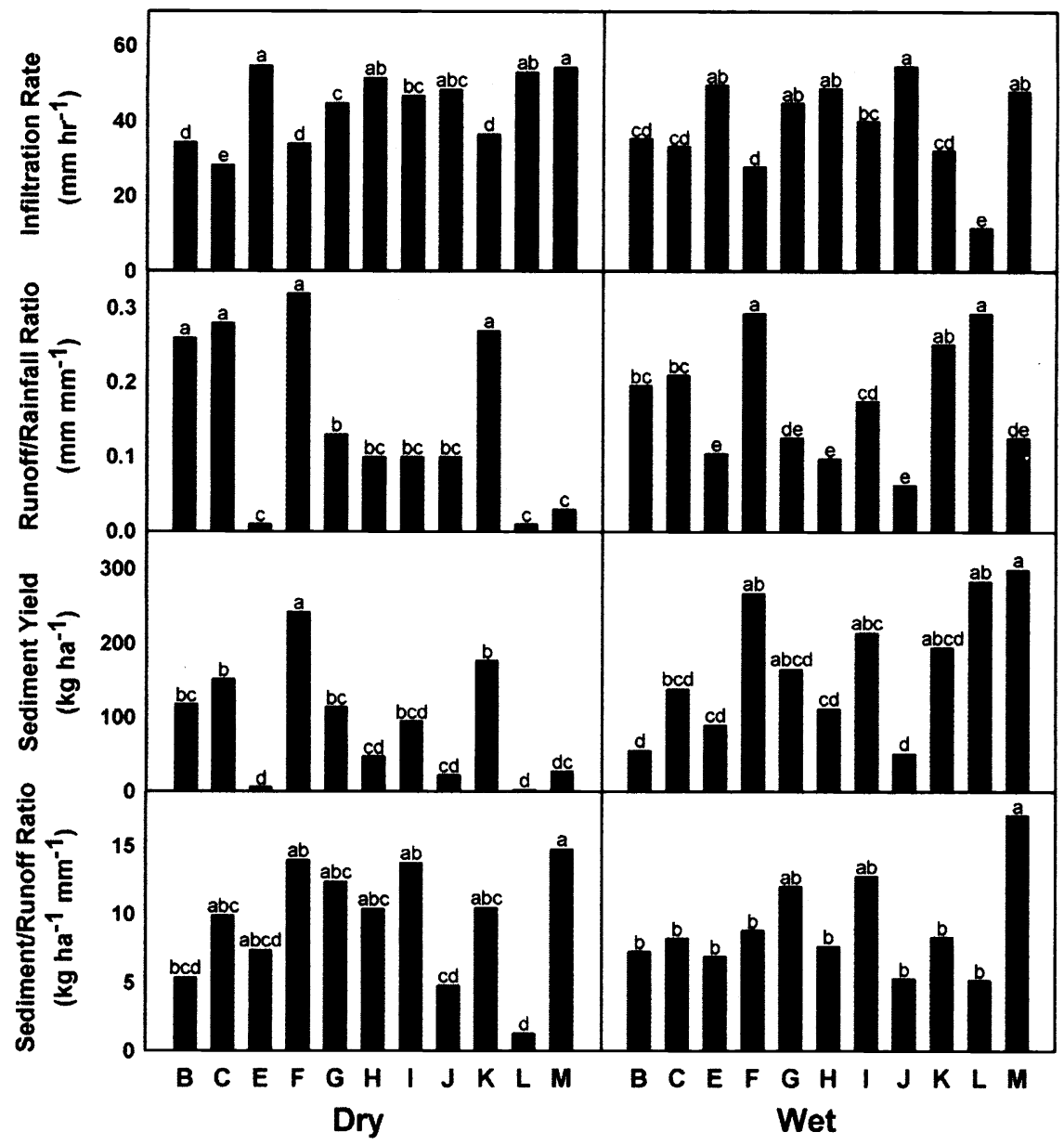

Fig. 2. Average a) terminal infiltration rate $\left(\mathrm{mm} \mathrm{hr}^{-1}\right)$, b) runoff/rainfall ratio $\left(\mathrm{mm} \mathrm{mm}^{-1}\right)$, c) total sediment $\left(\mathrm{kg} \mathrm{ha}^{-1}\right)$, and d) sediment/runoff ratio $\left(\mathrm{kg} \mathrm{ha}^{-1} \mathrm{~mm}^{-1}\right)$ for dry and wet runs for the 11 region/cover types studied. Values within dry or wet runs followed by a different letter are significantly different $(\mathbf{P}<0.10)$.

to how soil and vegetation properties affect infiltration and erosion processes.

\section{Across MLRAs}

The 11 rangeland soil-vegetation assemblages were analyzed to compare differences in hydrologic response across MLRAs, assuming that the vegetation states represented the typical range of vegetation and soil conditions found within MLRAs. For the majority of MLRAs, average terminal infiltration rates for the dry runs ranged from 40 to $55 \mathrm{~mm} \mathrm{hr}^{-1}$ (Fig. 2a). Slowest terminal infiltration rates occurred on the 3 grassland regions where shortgrass (or sodgrass) predominated. Wet terminal infiltration rates were slightly lower, but exhibited the same general pattern found in dry runs. The 1 exception, however, was annual grasslands. Dry terminal infiltration rates in annual grasslands were among the highest of the MLRAs, but the wet terminal infiltration rates were significantly lower than (percentage of rainfall lost to runoff, Fig. 2b) basically showed the same general patterns as those observed for terminal infiltration rates.

Colorado shortgrass prairie (F) had the greatest amount of total sediment under dry conditions, followed by the shortgrass site in Texas (C) and the Arizona shrubsteppe (K, Fig. 2c). Under wet antecedent soil moisture conditions, greatest sediment loss occurred from the sagebrush site in Utah (M), the annual grasslands, and the shortgrass prairie site in Colorado $(\mathrm{F})$. The tallgrass prairie regions in Nebraska (B) and Kansas (E), the mountain big sage- brush in Idaho $(\mathrm{J})$, and the mixed grass prairie in North Dakota $(\mathrm{H})$ had among the lowest total sediment production.

The sediment/runoff ratio provides a way to evaluate the erodibility of soil. Rangeland types with greater total sediment were not necessarily the MLRAs with the greatest soil erodibility. Under dry conditions, the Wyoming sagebrush site in Utah (M) ranked the highest for erodibility, and annual grasslands in California (L) the lowest (Fig. 2d). Sediment loading was greater from the 2 Wyoming sagebrush regions in Wyoming (I) and Utah (M) compared to the mountain big sagebrush region in Idaho $(\mathrm{J})$. Under wet conditions, there were few differences between sites in sediment loading. Many rangeland types were actually less erodible during the wet run. These rangelands may be detachment-limited, whereby the majority of erodible material had already been removed the previous day during the dry run.

When all sites were pooled together, infiltration and sediment production were not well correlated with any measured vegetation or soil characteristic $(\mathrm{R}<0.5)$. Forward multiple regression equations for infiltration and erosion variables were developed for both the dry (Table 9) and the wet runs (Table 10). Estimation equations containing 10 or more poorly correlated variables could explain only about $50 \%$ (dry condition) to $65 \%$ (wet condition) of the variation in infiltration and erosion occurring on all rangeland types. There was a slightly better fit for wet runs because of the removal of antecedent soil moisture as a source of variation. These regression equations were developed only to demonstrate why model dysfunction occurs when using these types of simplistic relationships (pooled data) for rangelands and should not be applied to management decisions.

\section{Discussion}

Infiltration and sediment production were differentially affected by vegetation and soil properties on rangeland types throughout the western United States. There were no consistent correlations or variables that affected infiltration or erosion on all sites. When all sites were pooled together, infiltration and sediment production were not well correlated $(\mathrm{R}<$ $0.5)$ with any measured vegetation or soil characteristic. The regression equations illustrate the poor fit that results when including all rangeland types. This type of 
Table 7. Correlation coefficients for various canopy cover, ground cover, above-ground biomass, root biomass, and soil characteristic variable classes for total (cumulative) infiltration, terminal infiltration rate, total (cumulative) sediment (log10), and sediment/runoff ratio (log 10) on short and annual grassland study sites. Only the highest correlated variable in each class and run is presented (some sites had few correlated variables). Correlations were performed for each state/region/cover type for both the dry and wet runs.

\begin{tabular}{|c|c|c|c|c|c|c|c|c|c|c|c|}
\hline \multirow[t]{2}{*}{$\begin{array}{c}\text { State, Region, } \\
\text { Cover Type }\end{array}$} & \multirow[t]{2}{*}{$\begin{array}{c}\text { Hydrologic/Erosion } \\
\text { Parameter }\end{array}$} & \multicolumn{2}{|c|}{$\begin{array}{c}\text { Canopy Cover } \\
\text { Variables }\end{array}$} & \multicolumn{2}{|c|}{$\begin{array}{c}\text { Ground Cover } \\
\text { Variables }^{2}\end{array}$} & \multicolumn{2}{|c|}{$\begin{array}{c}\text { Above-ground } \\
\text { Biomass Variables }^{3}\end{array}$} & \multicolumn{2}{|c|}{$\begin{array}{c}\text { Root Biomass } \\
\text { Variables }^{4}\end{array}$} & \multicolumn{2}{|c|}{$\begin{array}{c}\text { Soil } \\
\text { Variables }^{5} \\
\end{array}$} \\
\hline & & Dry & Wet & Dry & Wet & Dry & Wet & Dry & Wet & Dry & Wet \\
\hline \multirow{4}{*}{$\begin{array}{l}\text { Texas, } \\
\text { Shortgrass } \\
\text { prairie, Blue } \\
\text { grama- } \\
\text { buffalograss }\end{array}$} & $\begin{array}{l}\text { 1. Total Infiltration } \\
(\mathrm{mm})\end{array}$ & $\begin{array}{c}0.89 \\
(\text { st dead) }\end{array}$ & & $\begin{array}{c}-0.78 \\
\text { (bare) }\end{array}$ & & & & $\begin{array}{l}-0.72 \\
(\mathrm{sub})\end{array}$ & & $\begin{array}{c}0.94 \\
(\mathrm{ag} \mathrm{stab})\end{array}$ & $\begin{array}{c}0.85 \\
\text { (ag. stab) }\end{array}$ \\
\hline & $\begin{array}{l}\text { 2. Terminal Infiltration } \\
\text { Rate }\left(\mathrm{mm} \mathrm{hr}^{-1}\right)\end{array}$ & $\begin{array}{c}0.85 \\
\text { (st dead) }\end{array}$ & $\begin{array}{c}0.68 \\
\text { (forbs) }\end{array}$ & $\begin{array}{l}-0.67 \\
\text { (bare) }\end{array}$ & $\begin{array}{c}0.60 \\
\text { (basal) }\end{array}$ & $\begin{array}{c}0.66 \\
\text { (total std) }\end{array}$ & $\begin{array}{c}0.56 \\
\text { (total std) }\end{array}$ & $\begin{array}{l}-0.74 \\
\text { (sub) }\end{array}$ & $\begin{array}{l}-0.55 \\
(\mathrm{sub})\end{array}$ & $\begin{array}{c}0.94 \\
(\text { ag stab) }\end{array}$ & $\begin{array}{c}0.80 \\
\text { (ag stab) }\end{array}$ \\
\hline & $\begin{array}{l}\text { 3. Total Sediment } \\
\left(\mathrm{kg} \mathrm{ha}^{-1}\right)\end{array}$ & $\begin{array}{c}-0.81 \\
\text { (st dead) }\end{array}$ & $\begin{array}{c}-0.51 \\
\text { (st dead) }\end{array}$ & $\begin{array}{c}0.63 \\
\text { (bare) }\end{array}$ & & & & & & $\begin{array}{c}0.82 \\
\text { (sm sub) }\end{array}$ & \\
\hline & $\begin{array}{l}\text { 4. Sediment/Runoff } \\
\text { Ratio }\left(\mathrm{kg} \mathrm{ha}^{-1} \mathrm{~mm}^{-1}\right)\end{array}$ & & $\begin{array}{c}0.71 \\
\text { (forbs) }\end{array}$ & & $\begin{array}{l}-0.61 \\
\text { (bare) }\end{array}$ & & $\begin{array}{c}0.61 \\
\text { (forbs) }\end{array}$ & & $\begin{array}{c}-0.54 \\
\text { (average) }\end{array}$ & & $\begin{array}{c}-0.87 \\
\text { (BD ave) }\end{array}$ \\
\hline \multirow{4}{*}{$\begin{array}{l}\text { Colorado, } \\
\text { Mixedgrass } \\
\text { prairie, } \\
\text { Wheatgrass- } \\
\text { grama- } \\
\text { needlegrass }\end{array}$} & $\begin{array}{l}\text { 1. Total Infiltration } \\
(\mathrm{mm})\end{array}$ & & & & & & $\begin{array}{l}-0.51 \\
(\text { total })\end{array}$ & & & $\begin{array}{c}0.65 \\
\text { (sm sub) }\end{array}$ & \\
\hline & $\begin{array}{l}\text { 2. Terminal Infiltration } \\
\text { Rate }\left(\mathrm{mm} \mathrm{hr}^{-1}\right)\end{array}$ & & & & & $\begin{array}{c}-0.61 \\
\text { (forbs) }\end{array}$ & $\begin{array}{l}-0.56 \\
(\text { total })\end{array}$ & & & & \\
\hline & $\begin{array}{l}\text { 3. Total Sediment } \\
\left(\mathrm{kg} \mathrm{ha}^{-1}\right)\end{array}$ & $\begin{array}{l}-0.74 \\
\text { (grass) }\end{array}$ & & & & $\begin{array}{c}-0.51 \\
\text { (p grass) }\end{array}$ & & & & $\begin{array}{c}0.79 \\
\text { (sm surf) }\end{array}$ & $\begin{array}{c}-0.50 \\
(w B D s)\end{array}$ \\
\hline & $\begin{array}{l}\text { 4. Sediment/Runoff } \\
\text { Ratio }\left(\mathrm{kg} \mathrm{ha}^{-1} \mathrm{~mm}^{-1}\right)\end{array}$ & $\begin{array}{l}-0.69 \\
\text { (grass) }\end{array}$ & & & & $\begin{array}{c}0.57 \\
\text { (shrubs) }\end{array}$ & & & & $\begin{array}{c}0.78 \\
\text { (sm surf) }\end{array}$ & $\begin{array}{c}0.56 \\
\text { (sand s) }\end{array}$ \\
\hline \multirow{4}{*}{$\begin{array}{l}\text { California, } \\
\text { Annual } \\
\text { grassland, } \\
\text { Valley } \\
\text { grassland }\end{array}$} & $\begin{array}{l}\text { 1. Total Infiltration } \\
(\mathrm{mm})\end{array}$ & $\begin{array}{c}-0.86 \\
\text { (forbs) }\end{array}$ & & & $\begin{array}{c}-0.56 \\
\text { (litter) }\end{array}$ & $\begin{array}{c}-0.80 \\
\text { (total std) }\end{array}$ & $\begin{array}{c}-0.68 \\
(p \text { grass })\end{array}$ & $\begin{array}{l}0.74 \\
(\mathrm{sub})\end{array}$ & & $\begin{array}{c}0.81 \\
(\mathrm{ag} \mathrm{stab})\end{array}$ & \\
\hline & $\begin{array}{l}\text { 2. Terminal Infiltration } \\
\text { Rate }\left(\mathrm{mm} \mathrm{hr}^{-1}\right)\end{array}$ & & & & $\begin{array}{c}-0.51 \\
\text { (litter) }\end{array}$ & $\begin{array}{c}-0.68 \\
\text { (p grass) }\end{array}$ & & & & $\begin{array}{c}0.71 \\
\text { (sm surf) }\end{array}$ & \\
\hline & $\begin{array}{l}\text { 3. Total Sediment } \\
\left(\mathrm{kg} \mathrm{ha}^{-1}\right)\end{array}$ & & $\begin{array}{c}0.58 \\
\text { (forbs) }\end{array}$ & & & $\begin{array}{c}0.74 \\
(p \text { grass })\end{array}$ & $\begin{array}{c}0.71 \\
\text { (shrubs) }\end{array}$ & & & $\begin{array}{c}-0.74 \\
(\mathrm{sm} \mathrm{sub})\end{array}$ & $\begin{array}{c}0.63 \\
\text { (sm sub) }\end{array}$ \\
\hline & $\begin{array}{l}\text { 4. Sediment/Runoff } \\
\text { Ratio }\left(\mathrm{kg} \mathrm{ha}^{-1} \mathrm{~mm}^{-1}\right)\end{array}$ & & $\begin{array}{c}0.54 \\
\text { (forbs) }\end{array}$ & & $\begin{array}{c}-0.67 \\
\text { (litter) }\end{array}$ & $\begin{array}{c}0.54 \\
\text { (p grass) }\end{array}$ & $\begin{array}{l}-0.65 \\
\text { (litter) }\end{array}$ & & & $\begin{array}{c}-0.55 \\
\text { (sm sub) }\end{array}$ & $\begin{array}{c}-0.59 \\
(\mathrm{ag} \mathrm{stab})\end{array}$ \\
\hline
\end{tabular}

${ }^{1}$ st dead=standing dead

${ }^{2}$ bare $=$ bareground, basal $=$ basal cover

${ }^{3} \mathrm{p}$ grass=perennial grass, total=total yield, total $\mathrm{std}=$ total standing biomass

${ }^{4}$ average=average over soil sampled, sub=subsurface

${ }^{5}$ ag stab=aggregate stability, sm surf=surface antecedent soil moisture, sm sub=subsurface antecedent soil moisture, BD ave $=$ dry BD averaged

over soil sampled, wBD $s=$ surface wet $B D$, sand $s=$ surface sand content

pooled multiple regression equation is often used in the development of process models (Flanagan and Livingston 1995, Foster and Lane 1987), and the relatively low $\mathrm{R}^{2}$ value $(0.5$ to 0.65$)$ illustrates why infiltration and/or erosion estimates are inaccurate for some rangelands. Spaeth et al. (1996a) also found that universal equations representing a wide variety of rangeland plant communities were not as robust compared to equations develop for specific plant communities, due to the unique nature of plant communities. Weltz et al. (2000) also recommend partitioning rangelands according to soil functional units and developing nonlinear predictive equations to estimate infiltration for rangelands based on vegetation and soil characteristics.

Within rangeland types (within MLRA), there were generally an adequate array
(Franks et al. 1993) of vegetation and soil characteristics representing the range of typical vegetation states that helped point out the most important factors affecting infiltration and erosion on these soil types. In some cases, it was difficult to identify important variables due to highly variable runoff and erosion responses, especially on arid shrub-steppe rangelands. These rangelands are typified by high microsite variability in the spatial location of shrub coppices and interspaces, leading to greater variability between individual plots (Pierson et al. 1994, Blackburn 1975, Johnson and Gordon 1988).

Generally, dry-run infiltration rates ranged from 40 to $55 \mathrm{~mm} \mathrm{hr}^{-1}$ for most sites. Those sites with lower infiltration rates ( $<40 \mathrm{~mm} \mathrm{hr}^{-1}$ for the dry runs) were dominated by shortgrasses or sod-forming grasses and this is consistent with the liter- ature (Blackburn 1975, Wood and Blackburn 1981, Knight et al. 1984, Thurow et al. 1986,1988, Thurow 1991, Spaeth et al. 1996a).

Sediment production from all sites was less than $300 \mathrm{~kg} \mathrm{ha}^{-1}$. However, greater sediment/runoff ratios for some sites indicate a potential for more sediment loss should large, intense storms produce significant runoff. Under dry conditions, sites in Colorado (F3), Wyoming (G3, I1), North Dakota (H2), and Utah (M1) had greater potentials to produce higher sediment loads with large runoff events. Most of these sites tended to have higher bare soil exposure coupled with less litter and grass cover. Under wet conditions, sites in Wyoming (I1) and Utah (M1) still had a greater erosion potential from large runoff events. In addition, the Utah sagebrush site with brush management (M2) also had 
Table 8. Correlation coefficients for various canopy cover, ground cover, above-ground biomass, root biomass, and soil characteristic variable classes for total (cumulative) infiltration, terminal infiltration rate, total (cumulative) sediment (log10), and sediment/runoff ratio (log 10) on shrubsteppe study sites. Only the highest correlated variable in each class and run is presented (some sites had few correlated variables). Correlations were performed for each state/region/cover type for both the dry and wet runs.

\begin{tabular}{|c|c|c|c|c|c|c|c|c|c|c|c|}
\hline \multirow[t]{2}{*}{$\begin{array}{c}\text { State, Region, } \\
\text { Cover Type }\end{array}$} & \multirow[t]{2}{*}{$\begin{array}{c}\text { Hydrologic/Erosion } \\
\text { Parameter }\end{array}$} & \multicolumn{2}{|c|}{$\begin{array}{c}\text { Canopy Cover } \\
\text { Variables }\end{array}$} & \multicolumn{2}{|c|}{$\begin{array}{l}\text { Ground Cover } \\
\text { Variables }\end{array}$} & \multicolumn{2}{|c|}{$\begin{array}{c}\text { Above-ground } \\
\text { Biomass Variables }^{3}\end{array}$} & \multicolumn{2}{|c|}{$\begin{array}{c}\text { Root Biomass } \\
\text { Variables }^{4}\end{array}$} & \multicolumn{2}{|c|}{$\begin{array}{c}\text { Soil } \\
\text { Variables } \\
\end{array}$} \\
\hline & & Dry & Wet & Dry & Wet & Dry & Wet & Dry & Wet & Dry & Wet \\
\hline \multirow{4}{*}{$\begin{array}{l}\text { Wyoming, } \\
\text { Sage-grass- } \\
\text { shrub steppe, } \\
\text { Wyoming big } \\
\text { sagebrush }\end{array}$} & $\begin{array}{l}\text { 1. Total Infiltration } \\
(\mathrm{mm})\end{array}$ & $\begin{array}{c}-0.88 \\
\text { (forbs) }\end{array}$ & $\begin{array}{c}-0.65 \\
\text { (forbs) }\end{array}$ & $\begin{array}{c}0.58 \\
\text { (litter) }\end{array}$ & $\begin{array}{c}0.63 \\
\text { (litter) }\end{array}$ & $\begin{array}{c}0.63 \\
\text { (litter) }\end{array}$ & $\begin{array}{c}0.75 \\
\text { (a grass) }\end{array}$ & $\begin{array}{c}0.64 \\
\text { (int surf) }\end{array}$ & $\begin{array}{c}0.87 \\
\text { (int surf) }\end{array}$ & & $\begin{array}{c}-0.75 \\
\text { (sm sub) }\end{array}$ \\
\hline & $\begin{array}{l}\text { 2. Terminal Infiltration } \\
\text { Rate }\left(\mathrm{mm} \mathrm{hr}^{-1}\right)\end{array}$ & $\begin{array}{c}-0.88 \\
\text { (forbs) }\end{array}$ & $\begin{array}{c}-0.84 \\
\text { (forbs) }\end{array}$ & $\begin{array}{l}-0.68 \\
\text { (rock) }\end{array}$ & $\begin{array}{l}-0.68 \\
\text { (rock) }\end{array}$ & $\begin{array}{c}0.68 \\
\text { (litter) }\end{array}$ & $\begin{array}{c}0.81 \\
\text { (litter) }\end{array}$ & $\begin{array}{c}0.68 \\
\text { (int surf) }\end{array}$ & $\begin{array}{c}0.84 \\
\text { (int surf) }\end{array}$ & $\begin{array}{c}-0.50 \\
(\mathrm{BD} \mathrm{ss})\end{array}$ & $\begin{array}{c}-0.78 \\
\text { (sm sub) }\end{array}$ \\
\hline & $\begin{array}{l}\text { 3. Total Sediment } \\
\left(\mathrm{kg} \mathrm{ha}^{-1}\right)\end{array}$ & $\begin{array}{c}0.65 \\
\text { (forbs) }\end{array}$ & $\begin{array}{c}0.54 \\
\text { (forbs) }\end{array}$ & $\begin{array}{l}-0.63 \\
\text { (litter) }\end{array}$ & & $\begin{array}{c}-0.67 \\
\text { (litter) }\end{array}$ & $\begin{array}{c}-0.76 \\
\text { (litter) }\end{array}$ & $\begin{array}{c}-0.61 \\
\text { (int ave) }\end{array}$ & $\begin{array}{c}-0.63 \\
\text { (int ave) }\end{array}$ & $\begin{array}{c}0.53 \\
\text { (BD ave) }\end{array}$ & $\begin{array}{c}0.59 \\
\text { (sm sub) }\end{array}$ \\
\hline & $\begin{array}{l}\text { 4. Sediment/Runoff } \\
\text { Ratio }\left(\mathrm{kg} \mathrm{ha}^{-1} \mathrm{~mm}^{-1}\right)\end{array}$ & $\begin{array}{c}-0.54 \\
\text { (st dead) }\end{array}$ & & & & $\begin{array}{c}-0.63 \\
\text { (p grass) }\end{array}$ & & $\begin{array}{c}0.53 \\
\text { (average) }\end{array}$ & & $\begin{array}{c}0.54 \\
(\mathrm{BD} \mathrm{s})\end{array}$ & $\begin{array}{c}0.53 \\
(\mathrm{BD} \mathrm{s})\end{array}$ \\
\hline \multirow{4}{*}{$\begin{array}{l}\text { Idaho, } \\
\text { Sage-grass- } \\
\text { shrub steppe, } \\
\text { Mountain big } \\
\text { sagebrush }\end{array}$} & $\begin{array}{l}\text { 1. Total Infiltration } \\
(\mathrm{mm})\end{array}$ & & $\begin{array}{c}0.68 \\
\text { (shrubs) }\end{array}$ & & & & $\begin{array}{c}0.76 \\
\text { (forbs) }\end{array}$ & & & & $\begin{array}{c}-0.71 \\
\text { (sm surf) }\end{array}$ \\
\hline & $\begin{array}{l}\text { 2. Terminal Infiltration } \\
\text { Rate }\left(\mathrm{mm} \mathrm{hr}^{-1}\right)\end{array}$ & & & & $\begin{array}{l}-0.67 \\
\text { (bare) }\end{array}$ & & & & $\begin{array}{c}-0.59 \\
\text { (int sub) }\end{array}$ & & \\
\hline & $\begin{array}{l}\text { 3. Total Sediment } \\
\left(\mathrm{kg} \mathrm{ha}^{-1}\right)\end{array}$ & $\begin{array}{c}-0.56 \\
\text { (forbs) }\end{array}$ & $\begin{array}{c}0.56 \\
\text { (st dead) }\end{array}$ & & & & & & & & \\
\hline & $\begin{array}{l}\text { 4. Sediment/Runoff } \\
\text { Ratio }\left(\mathrm{kg} \mathrm{ha}^{-1} \mathrm{~mm}^{-1}\right)\end{array}$ & $\begin{array}{c}0.59 \\
\text { (st dead) }\end{array}$ & $\begin{array}{c}0.60 \\
\text { (st dead) }\end{array}$ & & & & & & & $\begin{array}{c}0.59 \\
\text { (sm sub) }\end{array}$ & \\
\hline \multirow{4}{*}{$\begin{array}{l}\text { Arizona, } \\
\text { Shrub steppe- } \\
\text { shortgrass, } \\
\text { Grama-galleta }\end{array}$} & $\begin{array}{l}\text { 1. Total Infiltration } \\
(\mathrm{mm})\end{array}$ & $\begin{array}{c}0.50 \\
\text { (forbs) }\end{array}$ & & $\begin{array}{c}-0.53 \\
\text { (basal) }\end{array}$ & & & $\begin{array}{r}-0.72 \\
(p \text { grass })\end{array}$ & & $\begin{array}{c}0.56 \\
\text { (int surf) }\end{array}$ & & $\begin{array}{c}0.55 \\
\text { (wBD ss) }\end{array}$ \\
\hline & $\begin{array}{l}\text { 2. Terminal Infiltration } \\
\text { Rate }\left(\mathrm{mm} \mathrm{hr}^{-1}\right)\end{array}$ & $\begin{array}{c}0.56 \\
\text { (forbs) }\end{array}$ & & $\begin{array}{c}-0.62 \\
\text { (basal) }\end{array}$ & $\begin{array}{c}0.51 \\
\text { (basal) }\end{array}$ & & $\begin{array}{c}-0.85 \\
\text { (p grass) }\end{array}$ & & $\begin{array}{c}0.70 \\
\text { (int surf) }\end{array}$ & & \\
\hline & $\begin{array}{l}\text { 3. Total Sediment } \\
\left(\mathrm{kg} \mathrm{ha}^{-1}\right)\end{array}$ & $\begin{array}{c}0.70 \\
\text { (st dead) }\end{array}$ & $\begin{array}{c}0.52 \\
\text { (st dead) }\end{array}$ & & & & $\begin{array}{c}-0.75 \\
\text { (a grass) }\end{array}$ & & $\begin{array}{c}-0.70 \\
\text { (int ave) }\end{array}$ & & \\
\hline & $\begin{array}{l}\text { 4. Sediment/Runoff } \\
\text { Ratio }\left(\mathrm{kg} \mathrm{ha}^{-1} \mathrm{~mm}^{-1}\right)\end{array}$ & & $\begin{array}{c}0.65 \\
\text { (st dead) }\end{array}$ & $\begin{array}{c}0.51 \\
\text { (crypts) }\end{array}$ & $\begin{array}{c}0.51 \\
\text { (crypts) }\end{array}$ & $\begin{array}{c}-0.53 \\
\text { (a grass) }\end{array}$ & $\begin{array}{c}-0.67 \\
\text { (a grass) }\end{array}$ & & $\begin{array}{c}-0.57 \\
\text { (int ave) }\end{array}$ & & \\
\hline \multirow{4}{*}{$\begin{array}{l}\text { Utah, } \\
\text { Shrub steppe, } \\
\text { Wyoming big } \\
\text { sagebrush }\end{array}$} & $\begin{array}{l}\text { 1. Total Infiltration } \\
(\mathrm{mm})\end{array}$ & & $\begin{array}{c}0.86 \\
\text { (grass) }\end{array}$ & $\begin{array}{c}0.52 \\
\text { (litter) }\end{array}$ & $\begin{array}{c}0.74 \\
\text { (litter) }\end{array}$ & & $\begin{array}{c}-0.75 \\
\text { (shrubs) }\end{array}$ & & $\begin{array}{c}0.73 \\
\text { (average) }\end{array}$ & & $\begin{array}{c}0.88 \\
\text { (BD ss) }\end{array}$ \\
\hline & $\begin{array}{l}\text { 2. Terminal Infiltration } \\
\text { Rate }\left(\mathrm{mm} \mathrm{hr}^{-1}\right)\end{array}$ & & & $\begin{array}{c}0.59 \\
\text { (litter) }\end{array}$ & $\begin{array}{c}0.53 \\
\text { (litter) }\end{array}$ & & $\begin{array}{c}0.55 \\
\text { (forbs) }\end{array}$ & & & & $\begin{array}{c}-0.67 \\
\text { (sm sub) }\end{array}$ \\
\hline & $\begin{array}{l}\text { 3. Total Sediment } \\
\left(\mathrm{kg} \mathrm{ha}^{-1}\right)\end{array}$ & $\begin{array}{c}-0.53 \\
\text { (grass) }\end{array}$ & & $\begin{array}{c}-0.89 \\
\text { (litter) }\end{array}$ & & & & $\begin{array}{c}-0.52 \\
\text { (average) }\end{array}$ & $\begin{array}{c}0.53 \\
\text { (int ave) }\end{array}$ & $\begin{array}{c}-0.64 \\
(\mathrm{BD} \mathrm{s})\end{array}$ & \\
\hline & $\begin{array}{l}\text { 4. Sediment/Runoff } \\
\text { Ratio }\left(\mathrm{kg} \mathrm{ha}^{-1} \mathrm{~mm}^{-1}\right)\end{array}$ & $\begin{array}{c}0.60 \\
\text { (shrubs) }\end{array}$ & & $\begin{array}{c}-0.76 \\
\text { (litter) }\end{array}$ & & $\begin{array}{c}0.53 \\
\text { (shrubs) }\end{array}$ & & $\begin{array}{c}-0.71 \\
\text { (int ave) }\end{array}$ & & $\begin{array}{c}0.62 \\
\text { (sm surf) }\end{array}$ & \\
\hline
\end{tabular}

\footnotetext{
st dead=standing dead

${ }^{2}$ crypts $=$ cryptogams, bare=bareground, basal=basal cover

${ }^{3} \mathrm{p}$ grass $=$ perennial grass, a grass $=$ annual grass

${ }^{4}$ average=average over soil sampled, int surf=interspace surface, int avg=interspace average

${ }^{5} \mathrm{sm}$ surf=surface antecedent soil moisture, sm sub=subsurface antecedent soil moisture, BD s=surface dry BD, BD ss=sub-surface dry BD, BD

avg=average dry $\mathrm{BD}$, wBD ss=sub-surface wet $\mathrm{BD}$
}

a sediment/runoff ratio exceeding $16.0 \mathrm{~kg}$ $\mathrm{ha}^{-1} \mathrm{~mm}^{-1}$, as did the prickly pear cactusdominated mixed grass prairie site in Wyoming (G1).

\section{Universal Assumptions}

The assumptions about hydrology and erosion relationships that were presented in the introduction did not hold true for all rangeland types studied. For every range- land site in this data set that reinforced the generalized assumptions, there was another rangeland site that refuted them.

\section{Rangeland ecological status/similarity index is directly related to hydrologic condition}

Greater ecological status or seral state was not always associated with improved hydrologic condition. For example, the cheatgrass site in Wyoming (G1) had similar infiltration to the late seral site dominated by native mid and short grasses (G3). Differences in bare ground and litter cover were more important than seral state in determining infiltration rates in this case. The same was true for the heavily grazed broomweed site (E1) in Kansas. This site had similar runoff and infiltration rates as compared to site E2 (dominated by mid and tall grasses) despite its early 
Table 9. Forward regression equations for dry run infiltration and erosion variables developed across all Major Land Resources Areas (MLRSs).

\begin{tabular}{|c|c|c|c|c|c|}
\hline Variable & $\begin{array}{c}\text { Terminal Infiltration } \\
\text { Rate }\end{array}$ & $\begin{array}{c}\text { Total } \\
\text { Infiltration }\end{array}$ & $\begin{array}{c}\text { Runoff/Rainfall } \\
\text { Ratio }\end{array}$ & $\begin{array}{c}\text { Total Sediment } \\
\left(\log _{10}\right)\end{array}$ & $\begin{array}{c}\text { Sediment/Runoff } \\
\text { Ratio }\left(\log _{10}\right)\end{array}$ \\
\hline & $\left(\mathrm{mm} \mathrm{hr}^{-1}\right)$ & $(\mathrm{mm})$ & $\left(\mathrm{mm} \mathrm{mm}^{-1}\right)$ & $\left(\mathrm{kg} \mathrm{ha}^{-1}\right)$ & $\left(\mathrm{kg} \mathrm{ha}^{-1} \mathrm{~mm}^{-1}\right)$ \\
\hline Intercept & 60.06 & 19.31 & -0.23 & 2.99 & 1.38 \\
\hline Variable 1 & 13.73 Grass Cover & 9.75 Grass Cover & -5.10 Cacti Cover & -51.80 Cacti Cover & -18.35 Cacti Cover \\
\hline Variable 2 & $\begin{array}{l}7.95 \text { Wet Surface } \\
\text { Bulk Density }\end{array}$ & $\begin{array}{l}\text { 354.25 Surface } \\
\text { Roughness }\end{array}$ & 0.53 Basal Cover & $\begin{array}{c}-0.63 \text { Dry Surface Bulk } \\
\text { Density }\end{array}$ & -2.89 Slope \\
\hline Variable 3 & $\begin{array}{l}-25.92 \text { Dry Sub- } \\
\text { surface Bulk Density }\end{array}$ & $\begin{array}{l}10.85 \text { Wet Surface } \\
\text { Bulk Density }\end{array}$ & $\begin{array}{l}\text { 0.36 Dry Sub-surface } \\
\text { Bulk Density }\end{array}$ & $\begin{array}{c}-0.0001 \text { Total Standing } \\
\text { Biomass }\end{array}$ & $\begin{array}{c}-0.0001 \text { Perennial Grass } \\
\text { Biomass }\end{array}$ \\
\hline Variable 4 & $\begin{array}{l}0.008 \text { Annual Grass } \\
\text { Biomass }\end{array}$ & $\begin{array}{c}0.002 \text { Total Standing } \\
\text { Biomass }\end{array}$ & $\begin{array}{l}-0.0001 \text { Annual } \\
\text { Grass Biomass }\end{array}$ & 0.00005 Root Biomass & 0.00003 Root Biomass \\
\hline Variable 5 & $\begin{array}{c}0.003 \text { Total Standing } \\
\text { Biomass }\end{array}$ & $\begin{array}{l}0.29 \text { Surface Cation } \\
\text { Exchange Capacity }\end{array}$ & $\begin{array}{c}-0.00004 \text { Total } \\
\text { Standing Biomass }\end{array}$ & $\begin{array}{l}-0.041 \text { Surface Cation } \\
\text { Exchange Capacity }\end{array}$ & $\begin{array}{l}-0.017 \text { Surface Cation } \\
\text { Exchange Capacity }\end{array}$ \\
\hline $\mathrm{R}^{2}$ & 0.33 & 0.38 & 0.36 & 0.47 & 0.30 \\
\hline $\begin{array}{l}\mathrm{R}^{2} \text { Maximum } \\
\text { (Complete Model, } \\
10+\text { variables) }\end{array}$ & 0.48 & 0.57 & 0.52 & 0.56 & 0.41 \\
\hline
\end{tabular}

seral state, apparently because of equivalent vegetation production, litter cover, and bare soil exposure.

The shortgrass/mixed grass prairie sites in Colorado had contrasting composition, production, and bare soil exposure and differed in their ecological status. Despite these differences, infiltration rate did not differ between the sites during either the dry or wet run. Spaeth et al. (1996b) also found that range ecological status may or may not be correlated to hydrologic condi- tion, depending on the structure and demography of the plant community.

\section{Sediment production is highly corre- lated with amount of infiltration/runoff}

Site characteristics affect infiltration and erosion processes in different ways. Therefore, sediment production may not be well correlated with amount of runoff. For example, total sediment for the wet run did not differ between vegetation states in the tallgrass prairie of Nebraska (B) or the shortgrass prairie of Texas (C) despite large differences in infiltration. Likewise, sites F1 and F3 in the shortgrass prairie of Colorado had similar runoff for the dry and wet runs, but site F1 had a lower sediment load than site F3. Less production of perennial grasses apparently led to greater sediment production from the heavily grazed F3 site. While California annual grassland sites (L1 and L2) did not differ in infiltration or runoff/rainfall ratio during

Table 10. Forward regression equations for wet run infiltration and erosion variables developed across all Major Land Resource Areas (MLRAs).

\begin{tabular}{|c|c|c|c|c|c|}
\hline Variable & $\begin{array}{l}\text { Terminal Infiltration } \\
\text { Rate }\end{array}$ & $\begin{array}{c}\text { Total } \\
\text { Infiltration }\end{array}$ & $\begin{array}{l}\text { Runoff/Rainfall } \\
\text { Ratio }\end{array}$ & $\begin{array}{c}\text { Total Sediment } \\
\left(\log _{10}\right)\end{array}$ & $\begin{array}{l}\text { Sediment/Runoff } \\
\text { Ratio }\left(\log _{10}\right)\end{array}$ \\
\hline & $\left(\mathrm{mm} \mathrm{hr}^{-1}\right)$ & $(\mathrm{mm})$ & $\left(\mathrm{mm} \mathrm{mm}^{-1}\right)$ & $\left(\mathrm{kg} \mathrm{ha}^{-1}\right)$ & $\left(\mathrm{kg} \mathrm{ha}^{-1} \mathrm{~mm}^{-1}\right)$ \\
\hline Intercept & 37.62 & 17.64 & 0.44 & 2.20 & 1.31 \\
\hline Variable 1 & $\begin{array}{c}\text { 60.85 Standing Dead } \\
\text { Cover }\end{array}$ & 32.72 Forb Cover & $\begin{array}{l}-0.59 \text { Standing Dead } \\
\text { Cover }\end{array}$ & $\begin{array}{l}-1.14 \text { Standing Dead } \\
\text { Cover }\end{array}$ & -0.42 Grass Cover \\
\hline Variable 2 & $\begin{array}{l}-0.96 \text { Surface Soil } \\
\text { Moisture }\end{array}$ & $\begin{array}{c}\text { 68.04 Standing Dead } \\
\text { Cover }\end{array}$ & $\begin{array}{l}0.015 \text { Surface Soil } \\
\text { Moisture }\end{array}$ & -30.88 Half-shrub Cover & $\begin{array}{l}0.50 \text { Cryptogam } \\
\text { Cover }\end{array}$ \\
\hline Variable 3 & $\begin{array}{l}\text { 0.37 Surface Sand } \\
\text { Content }\end{array}$ & $\begin{array}{c}-1.43 \text { Surface Soil } \\
\text { Moisture }\end{array}$ & $\begin{array}{c}-0.005 \text { Surface Sand } \\
\text { Content }\end{array}$ & $\begin{array}{l}\text { 0.021 Surface Soil } \\
\text { Moisture }\end{array}$ & $\begin{array}{c}-0.010 \text { Surface Soil } \\
\text { Moisture }\end{array}$ \\
\hline Variable 4 & $\begin{array}{l}\text {-19.66 Dry Subsurface } \\
\text { Bulk Density }\end{array}$ & $\begin{array}{l}0.26 \text { Surface Sand } \\
\text { Content }\end{array}$ & $\begin{array}{l}0.074 \text { Dry Subsurface } \\
\text { Bulk Density }\end{array}$ & $\begin{array}{c}-0.0003 \text { Perennial Grass } \\
\text { Biomass }\end{array}$ & $\begin{array}{l}0.00005 \text { Forb } \\
\text { Biomass }\end{array}$ \\
\hline Variable 5 & $\begin{array}{l}\text { 17.52 Subsurface } \\
\text { Organic Carbon }\end{array}$ & $\begin{array}{l}\text { 17.41 Subsurface } \\
\text { Organic Carbon }\end{array}$ & $\begin{array}{c}-0.24 \text { Subsurface } \\
\text { Organic Carbon }\end{array}$ & $\begin{array}{l}-0.46 \text { Subsurface } \\
\text { Organic Carbon }\end{array}$ & $\begin{array}{c}-0.00008 \text { Perennial } \\
\text { Grass Biomass }\end{array}$ \\
\hline $\mathrm{R}^{2}$ & 0.60 & 0.54 & 0.59 & 0.50 & 0.24 \\
\hline $\begin{array}{l}\mathrm{R}^{2} \text { Maximum } \\
\text { (Complete Model, } \\
10+\text { variables) }\end{array}$ & 0.66 & 0.69 & 0.69 & 0.56 & 0.29 \\
\hline
\end{tabular}


the wet run, L1 had twice the sediment yield as L2. L2 had significantly less exposed bare soil and more litter cover than L1. In this case, overland flow velocity was reduced on $\mathrm{L} 2$, thus producing less sediment compared to L1.

\section{Quantity of plant material and litter is positively correlated with infiltration}

Tallgrass prairie sites in Nebraska (B) represented highly contrasting vegetation states. Site B1 represented a Kentucky bluegrass-dandelion dominated site that had been subjected to heavy season-long use for many years. Site B2 was not grazed (hay meadow) and was dominated by primrose and mid and tall grasses with 3 times the productivity of $\mathrm{B} 1$. Cumulative infiltration and infiltration rate for both the dry and wet runs were negatively correlated with basal cover, but positively correlated with total yield. Litter cover and/or biomass were not strongly correlated with infiltration on 5 out of 11 rangeland sites, and total biomass was a correlate on only 4 rangeland types. Individual plant species or overall species composition and the structural component of the plant community may be more important than total cover or biomass (Spaeth et al. 1996a, 1996b). Root morphology, plant growth form and architecture, spatial pattern of plants, soil chemical or physical factors, soil crusts, and micro flora, etc. are attributes of the plant community (associated with individual species or community structure) which can influence infiltration and erosion, but are difficult to measure (Spaeth et al. 1996a).

\section{Brush management alters hydrology and erosion}

The Wyoming big sagebrush sites in Wyoming (I) differed in grass and shrub cover due to brush control on site I2 (prescribed burn 3 years prior to study). Infiltration differed slightly for both the dry and wet runs, but total sediment was equivalent for both sites (I1, I2). The mountain big sagebrush sites in Idaho $(\mathrm{J})$ also differed primarily in shrub and grass cover due to brush control on site $\mathbf{J} 2$ that reduced shrub cover from $40 \%$ to about $5 \%$ (chemical control eight years prior to study). Yet infiltration rates and total sediment did not differ between the 2 sites ( $\mathrm{J} 1$, $\mathrm{J} 2$ ). Both sites had relatively high surface and subsurface organic carbon contents and surface roughness that probably contributed to the good infiltration rates measured on both sites. The Wyoming big sagebrush sites in Utah (M) represented differences between an undisturbed site
(M1) and a site that had a history of brush control (chemical control 3 years prior to study)(M2). There were no differences in infiltration rate for the dry or wet run, and only the dry run showed slight differences in total sediment production.

High variability in hydrologic response among plots within a site in some cases made it difficult to discern true differences between vegetation states. This was especially true on the range sites where undisturbed shrub and shrub-converted sites were compared. Soil characteristics and structure associated with shrub-interspace zonation may still exist after conversion (Hester et al. 1997), resulting in similar hydrologic response despite differences in shrub cover. Also, greater variability in soil and vegetation characteristics due to this zonation makes predictive relationships more difficult to ascertain (Pierson et al. 1994).

\section{Vegetation characteristics dominate rangeland hydrology response}

Annual grasslands in California (L) had the highest infiltration rates under dry soil conditions, but wet infiltration rates were the slowest measured on any site. These clayey soils (high smectite content) had sub-surface cracking under dry conditions, leading to preferential flow. Once wet, these cracks closed and infiltration rates measured were the lowest for the wet runs. The L1 and L2 sites varied considerably in the amount of bare ground, litter cover, grass cover and biomass, total standing biomass, and litter biomass, yet infiltration and erosion differed very little between the two vegetation states. Vegetation characteristics such as total standing biomass and litter were actually negatively correlated with infiltration. Soil properties, rather than vegetation characteristics, were the predominant factor controlling hydrologic response on these annual grasslands.

\section{Implications}

The examples presented above emphasize the difficulty in the "one size fits all" modeling approach in developing universal algorithms to include all rangeland types. While the amount of vegetation and corresponding litter have been found to be the most correlated variables with infiltration (Branson et al. 1981), others have found that the relationship between plant cover/litter and infiltration rates is not well established on semiarid rangelands (Gifford 1968, Blackburn 1975). The amount (biomass and cover) and type of vegetation and litter, canopy structure, rooting patterns, soil physical properties, small-scale spatial variability, bare soil exposure, potential for soil crusting, slope gradient, consumptive water use, and seasonal dynamics can affect hydrology and erosion to varying degrees depending on the plant community type (Rauzi 1960, Johnson 1962, Branson and Owens 1970, Meeuwig 1970, Tromble et al. 1974, Blackburn 1975, Davis and Pase 1977, Branson et al. 1981, Wood and Blackburn 1981, Hibbert 1983, Knight et al. 1984, Thurow et al. 1986, 1988 Johnson and Gordon 1988, Wilcox et al. 1988, Holmstead 1989, Hicks et al. 1990, Thurow 1991, Pierson et al. 1994, Spaeth et al. 1996a, 1996b, Weltz et al. 1998). The NRST data set provides some information as to the degree of influence that various soil and vegetation variables have on infiltration and erosion. However, it also emphasizes the difficulty in determining hydrologic relationships on semiarid rangelands, where infiltration rates are determined from a myriad group of factors that are different (or differ in importance) depending on rangeland type and site conditions.

There are quite a few generalizations or assumptions about the relationships between rangeland soil and vegetation characteristics and infiltration and erosion, and there are examples presented in the NRST dataset that both confirm and refute these generalizations. The regression equations presented highlight the poor fit that results when including all rangeland types. Previous models that have attempted to characterize rangeland infiltration and erosion using similar generalized or generic models/algorithms have not performed well because of this complex interaction of factors that differ from one soilvegetation assemblage to the next (Pierson et al. 2001). A new paradigm to organize rangeland communities into "functional" units according to similarity in relationships and responses could aid in the development of better models to more accurately predict infiltration and erosion on rangelands.

\section{Literature Cited}

Alberts, E.E., M.A. Nearing, M.A. Weltz, L.M. Risse, F.B. Pierson, X.C. Zhang, J.M. Laflen, and J.R. Simanton. 1995. Soil Component (Chapter 7). p. 7.1-7.47. In: D.C. Flanagan and S. J. Livingston (Editors). USDA-Water erosion prediction project (WEPP). NSERL Report No. 11. USDAARS, Nat. Soil Erosion Res. Lab., West Lafayette, Ind. 
Bedunah, D.J. and R.E. Sosebee. 1985. Influence of site manipulation on infiltration rates of a depleted west Texas range site. J. Range Manage. 38:200-205.

Blackburn, W.H. 1975. Factors influencing infiltration and sediment production of semiarid rangelands in Nevada. Water Resour. Res., 11:929-937.

Blackburn, W.H. and C.M. Skau. 1974. Infiltration rates and sediment production of selected plant communities and soils of Nevada. J. Range Manage. 27:476-480.

Blake, R.G. and K.H. Hartge. 1986. Bulk density. P. 363-376. In: A. Klute (ed.). Methods of soil analysis. Part I. Physical and mineralogical methods. Agron. Ser. 9, Amer. Soc. Agr., Soil Sci. Soc. Amer., Madison, Wisc.

Branson, F.A. and J.B. Owens. 1970. Plant cover, runoff, and sediment yield relationships on Mancos Shale in western Colorado. Water Resour. Res. 6:783-790.

Branson, F.A., G.F. Gifford, K.G. Renard, and R.F. Hadley. 1981. Rangeland hydrology, 2nd edition. Kendall/Hunt publishing Co., Dubuque, Ia. pp. 340.

Buckhouse, J.C. 1984. Vegetation and soil indicators to hydrologic potential. Rangelands 6:206-209.

Carlson, D.H., T.L. Thurow, R.W. Knight, and R.K. Heitschmidt. 1990. Effect of honey mesquite on the water balance of Texas rolling plains rangeland. J. Range Manage. 43:491-496.

Davis, E.A. and C.P. Pase. 1977. Root systems of shrub live oak: implications for water yield in Arizona chaparral. J. Soil Water Conserv. 32:174-180.

Ellison, W.D. 1949. Protecting the land against the raindrop's blast. Sci. Monthy 68:241-251.

Flanagan, D.C. and S. J. Livingston (Editors). 1995. USDA-Water erosion prediction project (WEPP). NSERL Report No. 11. USDA-ARS, Nat. Soil Erosion Res. Lab., West Lafayette, Ind.

Foster, G.R. and L.J. Lane (eds.). 1987. User Requirements. USDA-Water Erosion Prediction Project (WEPP) Draft 6.3. NSERL Report No. 1. USDA-ARS, Nat. Soil Erosion Res. Lab., West Lafayette, Ind.

Franks, C.D., L. C. Brockmann, M. Whited, and S.W. Waltman. 1993. Using the benchmark site selection process to enhance modeling. In: Abstracts, Predicting Rangeland Hydrologic and Erosion Processes Symposium. The $46^{\text {th }}$ Annual Meeting of the Soc. for Range Manage., Albuquerque, N.M., Febr. 14-19, 1993.

Franks, C.D., F.B. Pierson, A.G. Mendenhall, K.E. Spaeth, and M. Weltz. 1998. Interagency rangeland water erosion project report and state data summaries. NWRC Report No. 98-1. USDA-ARS, Northw. Watershed Res. Center, Boise, Ida.

Gifford, F.G. 1968. Rangeland watershed management-a review. Univ. Nevada Agr. Exp. Station, Paper R52.
Gifford, G.F. 1985. Cover allocation in rangeland watershed management (a review), $\mathrm{p}$. 23-31. In: Proc. Watershed Management in the Eighties (eds. E.B. Jones and T.J. Ward), Amer. Soc. of Civil Engineers, New York, N.Y.

Hester, J.W., T.L. Thurow, and C. A. Taylor. 1997. Hydrologic characteristics of vegetation types as affected by prescribed burning. J. Range Manage. 50:199-204.

Hibbert, A.R. 1983. Water yield improvement potential by vegetation management on western rangelands. Water Resour. Bull 19:375-381.

Hicks, R.A., D.D. Briske, C.A. Call, and R.J. Ansley. 1990. Co-existence of a perennial $C_{3}$ bunchgrass in a $\mathrm{C}^{4}$ dominated grassland: an evaluation of gas exchange characteristics. Photosynthetica 24:63-74.

Holmstead, G.L. 1989. Water-use and growth of three $\mathrm{C}_{4}$ bunchgrasses: evaluation under field and controlled environment conditions. M.S. Thesis, Texas A\&M Univer., College Station, Tex.

Johnson, A. 1962. Effects of grazing intensity and cover on the water intake rate of Fescue grassland. J. Range Manage. 15:79-82.

Johnson, C.W. and N.D. Gordon. 1988. Runoff and erosion from rainfall simulation plots on sagebrush rangeland. Trans. Amer. Soc. Agr. Eng. 31:421-427.

Knight, R.W., W.H. Blackburn, and L.B. Merrill. 1984. Characteristics of oak mottes, Edwards Plateau, Texas. J. Range Manage. 37:534-537.

Meeuwig, R.O. 1970. Infiltration and soil erosion as influenced by vegetation and soil in northern Utah. J. Range Manage. 23:185-188.

Mueller-Dombois, D. and E. Ellenberg. 1974. Aims and methods of vegetation ecology. John Wiley and Sons, New York, N.Y.

Osborn, B. 1952. Storing rainfall at the grass roots. J. Range Manage. 5:408-414.

Pierson, F.B., D.H. Carlson, and K. E. Spaeth. 2001. A Process-Based Hydrology Submodel Dynamically Linked to the Plant Component of the SPUR Model . Eco. Mod. 141:241-260.

Pierson, F.B., S.S. Van Vactor, W.H. Blackburn, and J.C. Wood. 1994. Incorporating small-scale spatial variability into predictions of hydrologic response on sagebrush rangelands. Special Publication 38:23-34. Soil Sci. Soc. of Amer., Madison, Wisc.

Rauzi, F. 1960. Water intake studies on range soils at 3 locations in the Northern Plains. J. Range Manage. 13:179-184.

SAS Institute Inc. 1999. SAS/STAT users guide, Version 6.12. SAS Institute Inc., Cary, N.C.

Simanton, J.R., M.A. Weltz, and H.D. Larsen. 1991. Rangeland experiments to parameterize the water erosion prediction project model: Vegetation canopy cover effects. J. Range Manage. 44:276-282.
Simanton, J.R., T.L. West, M.A. Weltz, and W.G. Wingate. 1987. Rangeland experiments for water erosion prediction project. Trans. Amer. Soc. Agr. Eng. Paper No. 872545. St. Joseph, Mich.

Spaeth, K.E., F.B. Pierson, M.A. Weltz, and J.B. Awang. 1996a. Gradient analysis of infiltration and environmental variables as related to rangeland vegetation. Trans. Amer. Soc. Agr. Eng. 39:67-77.

Spaeth, K.E., T.L. Thurow, W.H. Blackburn, and F.B. Pierson. 1996b. Ecological dynamics and management effects on rangeland hydrologic processes, $p$. 25-51.In: K.E. Spaeth, F.B. Pierson, M.A. Weltz, and G. Hendricks (eds.). Grazingland hydrology issues: Perspectives for the $21^{\text {st }}$ century. Soc. for Range Manage., Denver, Colo.

Stoddart, L.A., A.D. Smith, and T.W. Box. 1975. Range management. McGraw-Hill Book Company, New York, N.Y. pp. 532.

Swanson, N.P. 1965. Rotating-boom rainfall simulator. Trans. Amer. Soc. Agr. Eng. 8:71-72.

Thurow, T.L. 1991. Hydrology and erosion, p. 141-160. In: R.K. Heitschmidt and J.W. Stuth (Editors). Grazing management: an ecological perspective. Timber Press Inc., Portland, Ore.

Thurow, T.L., W.H. Blackburn, and C.A. Taylor. 1986. Hydrologic characteristics of vegetation types as affected by livestock grazing systems, Edwards Plateau, Texas. J. Range Manage. 39:505-509.

Thurow, T.L., W.H. Blackburn, and C.A. Taylor. 1988. Infiltration and interrill erosion responses to selected livestock grazing strategies, Edwards Plateau, Texas. J. Range Manage. 41:296-302.

Tromble, J.M., K.G. Renard, and A.P. Thatcher. 1974. Infiltration for 3 rangeland soil-vegetation complexes. J. Range Manage. $27: 318-321$

United States Department of AgricultureNatural Resources Conservation Service (USDA-NRCS). 1997. National Range and Pasture Handbook. Grazing Lands Technology Institute. Fort Worth, Tex.

Weltz, L., G. Frasier, and M. Weltz. 2000. Hydrologic response of shortgrass prairie ecosystems. J. Range Manage. 53:403-409.

Weltz, M.A., M.R. Kidwell, and H.D. Fox. 1998. Influence of abiotic and biotic factors in measuring and modeling soil erosion on rangelands: State of knowledge. J. Range Manage. 51:482-495.

Wilcox, B.P., M.K. Wood, and J.H. Tromble. 1988. Factors influencing infiltrability of semiarid mountain slopes. J. Range Manage. 41:197-206.

Wood, M.K. and W.H. Blackburn. 1981. Grazing systems: their influence on infiltration rates in the Rolling Plains of Texas. J. Range Manage. 34:331-335. 